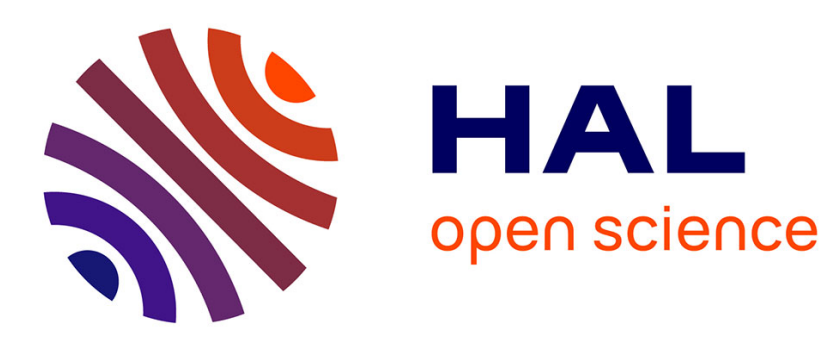

\title{
De l'intégration interne du système d'information à l'intégration du système d'information de la chaîne logistique
}

François de Corbière, Frantz Rowe, François-Charles Wolff

\section{- To cite this version:}

François de Corbière, Frantz Rowe, François-Charles Wolff. De l'intégration interne du système d'information à l'intégration du système d'information de la chaîne logistique. 2012. hal-00671507

\section{HAL Id: hal-00671507 \\ https://hal.science/hal-00671507}

Preprint submitted on 17 Feb 2012

HAL is a multi-disciplinary open access archive for the deposit and dissemination of scientific research documents, whether they are published or not. The documents may come from teaching and research institutions in France or abroad, or from public or private research centers.
L'archive ouverte pluridisciplinaire HAL, est destinée au dépôt et à la diffusion de documents scientifiques de niveau recherche, publiés ou non, émanant des établissements d'enseignement et de recherche français ou étrangers, des laboratoires publics ou privés. 
EA 4272

\title{
De l'intégration interne du système d'information à l'intégration du système d'information de la chaîne logistique
}

\author{
François de Corbière* \\ Frantz Rowe** \\ François-Charles Wolff ***
}

Laboratoire d'Economie et de Management Nantes-Atlantique Université de Nantes

Chemin de la Censive du Tertre - BP 52231

44322 Nantes cedex 3 - France

www.univ-nantes.fr/iemn-iae/recherche

Tél. +33 (0)2 40141717 - Fax +33 (0)2 40141749 


\title{
De l'intégration interne du système d'information à l'intégration du système d'information de la chaîne logistique ${ }^{\#}$
}

\author{
Frantz Rowe $^{*}$, François-Charles Wolff ${ }^{* *}$, François de Corbière ${ }^{* * *}$
}

\begin{abstract}
Résumé :
L'objectif de ce papier est de vérifier s'il existe une relation entre l'intégration des SI en interne et en externe. L'analyse se base sur la littérature liant intégration interne et externe et notamment sur le schéma proposé par Venkatraman présentant les phases d'évolution de l'entreprise. A partir de l'enquête COI-TIC, des indicateurs synthétiques de l'intégration des SI en interne et de l'intégration des SI de la chaîne logistique sont construits. Leurs facteurs explicatifs sont analysés sur un échantillon de 9721 entreprises. Les résultats montrent sur ce large échantillon que l'intégration interne du SI vient accroître l'intégration du SI de la supply chain. Parmi les technologies utilisées en interne, les ERP ont la plus grande influence sur l'intégration interne et constituent le meilleur prédicteur de l'intégration du SI de la chaîne logistique. Les secteurs d'activité offrent néanmoins des écarts importants entre l'intégration des SI en interne et en externe.
\end{abstract}

Mots clefs : Intégration interne, intégration externe, ERP, corrélation et causalité, variables de contingence, chaîne logistique.

\begin{abstract}
:
The paper aims at testing if there exists a relationship between internal IS integration and external IS integration. The analysis is based on the literature that links internal and external integration and proposes a set of contingency variables. Contrary to operational research literature, Venkatraman's scheme of evolutionary stages emphasizes a disconnect between internal and external. Using data from the COI-TIC survey, synthetic indicators of internal IS integration and supply chain IS integration are built and their explanatory factors are analyzed on a sample of 9721 firms. Among technologies used internally, ERP have the greatest influence on internal IS integration and are the best predictor of supply chain IS integration. Economic sectors show discrepancies between internal and external IS integration.
\end{abstract}

Key-words: Internal IS integration, external IS integration, ERP, correlation and causality, contingency variables, supply chain.

\footnotetext{
\# Cette recherche s'inscrit dans le cadre du projet scientifique OLASI (Optimisation des réseaux Logistiques, stratégie Achats et Systèmes d'Information: entreprise étendue et performance), financé par la Région des Pays de La Loire et soutenu par les pôles de compétitivité MC2 et Nov@log. Cette communication a fait l'objet d'une présentation au 16ème colloque annuel de l'Association Information et Management (Saint-Denis de la Réunion, 25-27 mai 2011).

${ }^{*}$ Professeur en Sciences de Gestion, LEMNA, Université de Nantes.

E-mail: frantz.rowe@univ-nantes.fr

${ }^{* *}$ Professeur en Sciences Economiques, LEMNA, Université de Nantes et INED, Paris, France.

E-mail: francois.wolff@univ-nantes.fr http://www.sc-eco.univ-nantes.fr/ fcwolff

${ }^{* * *}$ Maître de Conférences, LEMNA, Ecole des Mines de Nantes. E-mail: francois.de-corbiere@mines-nantes.fr
} 


\section{Introduction}

L'intégration des systèmes d'information, définie comme un état cohérent des données supporté par des systèmes interdépendants (Reix et al., 2011) est un thème majeur tant pour le milieu académique que pour les praticiens. Cela tient à la fois à des raisons culturelles et politiques. L'informaticien rêve de l'outil qui serait le parfait couteau Suisse du traitement de l'information. Il passe une bonne partie de son temps à gérer des problèmes de compatibilité de standards, de versions et d'outils, et son travail est plus aisé quand les outils sont intégrés. D'un point de vue politique, la tentation du contrôle animant les organisations et les directions générales des entreprises les pousse à mettre en œuvre des systèmes de plus en plus intégrés lorsqu'elles estiment que les risques induits ne les rendent pas trop vulnérables (Bidan et Rowe, 2004). Dans ce contexte, après la diffusion au sein des entreprises de technologies intégratives et notamment des progiciels intégrés de gestion (ERP en anglais), la question de l'intégration se pose pour les opérations interorganisationnelles (Bidan, 2004 ; de Corbière et Geffroy, 2009; Markus, 2000). D'ailleurs, l'intégration des SI a été récemment définie comme "l'unification des processus, systèmes et/ou données depuis de multiples systèmes informatisés, et ce pas nécessairement dans une seule organisation »(Seddon et al., 2010).

En même temps, le problème évolue. Il s'agit désormais moins d'intégrer des systèmes de façon complète puisque chaque organisation ne peut prétendre avoir en son sein tout le système d'information de ses clients et fournisseurs, sauf à aller vers une véritable intégration économique qui passe par des opérations capitalistiques. En dépit de ces réserves, les entreprises cherchent à intégrer au moins partiellement leurs systèmes pour constituer des systèmes interorganisationnels, notamment lorsqu'il s'agit d'opérations régulières telles que les commandes, les expéditions, les réceptions et plus généralement les opérations logistiques (Rai et al., 2006). Face à cette demande, les éditeurs de logiciels proposent depuis des années des solutions, à commencer par les logiciels de traduction pour mettre en œuvre l'EDI (Echange de Données Informatisées). Des applications plus récentes ont émergé sous la forme de modules ERP ou bien de progiciels spécialisés dédiés à la gestion de la chaîne logistique. On parle alors d'applications ERP II ou SCM (Supply Chain Management) (Beatty et Williams, 2006 ; Daniel et White, 2005 ; Koh et al., 2011).

La diffusion de l'informatisation et le degré d'intégration des SI en France constituent un thème classique et d'actualité autant pour les patriciens que pour les chercheurs. Dans cet article, nous étudions le lien entre l'intégration interne du SI et l'intégration du SI de la chaîne logistique. Venkatraman (1994) propose une vision schématique du changement dans l'entreprise suggérant que l'informatisation de l'entreprise évolue d'un niveau intraorganisationnel vers les niveaux interorganisationnels pour enfin arriver à un stade d'entreprise en réseau. En théorie, cela permettrait d'arriver à des objectifs de création de valeur et de transformation d'ordre supérieur. On peut toutefois se demander si les entreprises déployant un système d'information vers leurs clients et fournisseurs ont besoin d'un système véritablement intégré et si les déterminants de l'intégration interne du SI sont également explicatifs de l'intégration du SI de la supply chain. Pour répondre à cette question, nous mobilisons des données issues de l'enquête COI sur un échantillon de 9721 entreprises ayant toutes les fonctions classiques. Cet échantillon représentatif de la population des entreprises en France rend les résultats de cette recherche particulièrement intéressants en raison de leur forte validité externe. Si l'on ne peut prétendre comprendre aussi finement le phénomène de l'intégration que certaines études de cas, l'échantillon analysé permet en revanche de traiter à la fois du lien entre les deux concepts d'intégration et des conditions de contingence. 
Suite à une revue de littérature sur l'intégration du SI et ses principaux facteurs d'influence, la partie méthodologique de cet article présente l'analyse économétrique que nous faisons de l'intégration des SI par l'exploitation de l'enquête COI-TIC. Pour cela, des indicateurs synthétiques de l'intégration interne du SI et de l'intégration du SI de la chaîne logistique sont construits. Les résultats des facteurs d'influence sur les deux indicateurs sont mis en exergue avant de rendre compte des liens de corrélation et de causalité entre les deux concepts d'intégration. La discussion et la conclusion mettent en perspective ces résultats obtenus sur un échantillon très important des entreprises françaises.

\section{Revue de la littérature}

\subsection{La couverture et l'intégration des systèmes d'information : vers l'entreprise étendue}

Venkatraman (1994) distingue plusieurs étapes d'évolution de l'entreprise, commençant par l'informatisation et l'exploitation locale de telle ou telle innovation informatique au sein de telle ou telle fonction, puis passant à l'étape de l'intégration intra-organisationnelle (figure 1). Le passage à l'étape suivante indique une rupture forte puisque l'on est censé alors s'engager dans des étapes révolutionnaires. Il distingue alors trois niveaux de la transformation des affaires : la redéfinition des processus, la redéfinition du réseau, puis enfin la redéfinition de l'activité. S'il est clair dans son schéma que l'évolution se situe à un niveau intraorganisationnel, ses commentaires indiquent bien que l'interconnectivité interorganisationnelle ne suffit pas pour passer aux phases ultérieures : redéfinition est le mot clé dans la révolution!

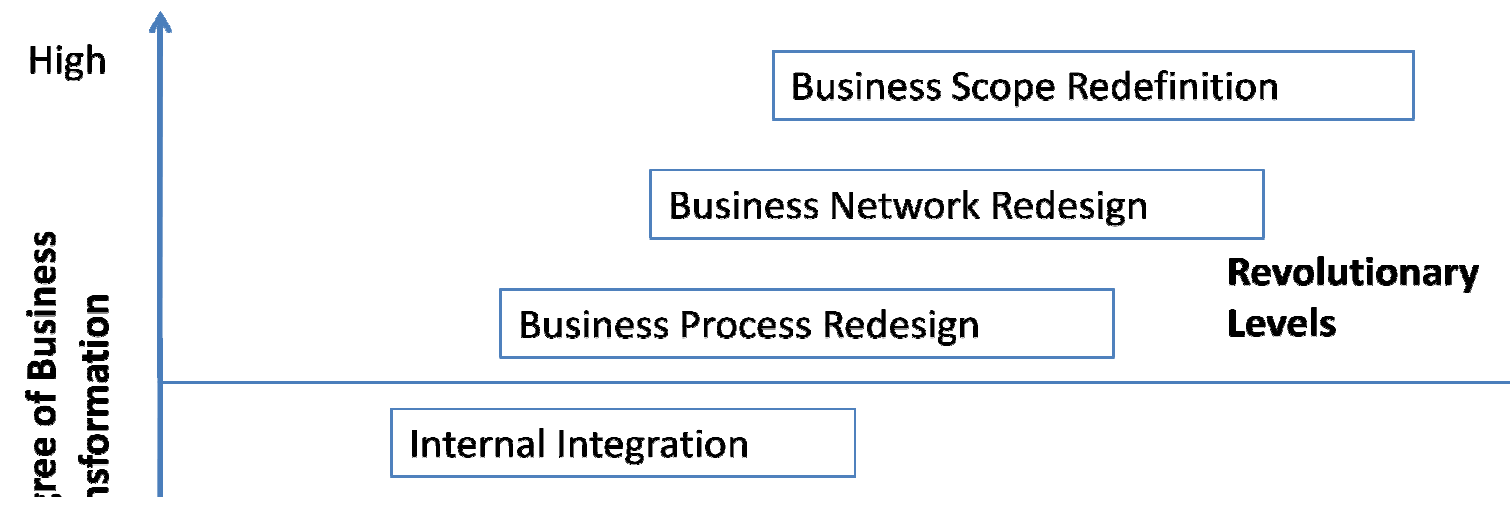

Figure 1 : Schéma d'évolution de Venkatraman (1994)

Dans ce contexte, la constitution d'une entreprise étendue informatisée est le produit conjoint de changements organisationnels significatifs et de systèmes d'information intégrés. Notons au passage que les systèmes d'information intégrés dépendent eux-mêmes fondamentalement de tels changements et du périmètre couvert (El Amrani et al., 2006). Autrement dit, 
l'extension du périmètre organisationnel, quel qu'il soit, repose sur l'intégration des systèmes internes et sur des changements organisationnels profonds (Venkatraman, 1994).

Dans ce cadre, une récente recherche synthétise les bénéfices organisationnels issus de l'intégration par les systèmes d'entreprises (Seddon et al., 2010). L'intégration permet tout d'abord de réduire considérablement les pertes issues de travaux superflus liés à la résolution de problèmes concernant la qualité de l'information et la compatibilité entre les sources des données. De plus, l'intégration des données constitue une base solide pour l'optimisation des processus et la prise de décision. Enfin, avoir une interface commune pour plusieurs applications offre aussi une appropriation et une utilisation plus aisées par les utilisateurs finaux. Globalement, que ce soit pour les ERP ou d'autres formes de systèmes d'entreprises, la transformation organisationnelle induite par l'intégration apporte d'indéniables bénéfices par la standardisation des données et des processus pour les opérations génériques et interdépendantes de l'entreprise. Toutefois, elle nuit aux opérations nécessitant de la différenciation (Gattiker et Goodhue, 2005). Dans cette perspective, Strong et Volkoff (2010) identifient différents domaines dans lesquels on peut constater un non alignement entre l'organisation et son système intégré.

Si l'on peut retrouver cette question fondamentale entre interdépendances et différenciation au niveau des opérations interorganisationnelles, il n'en reste pas moins que le principe et les modalités d'intégration se déplacent vers les relations de l'entreprise avec ses partenaires (Markus, 2000). En particulier, les entreprises ont historiquement cherché avant tout à développer l'intégration externe de leurs systèmes d'information sur les opérations logistiques régulières telles que les commandes, les expéditions ou les réceptions. La recherche sur l'intégration de la supply chain met notamment en évidence l'intrication forte entre les flux physiques et informationnels pour les aspects transactionnels de la relation (Livolsi et FabbeCostes, 2004 ; Patnayakuni et al., 2007 ; Prajogo and Ohlager, 2012). C'est notamment dans ce cadre que l'EDI a favorisé l'intégration du SI de la supply chain (Srinivasan et al., 1994 ; Truman, 2000 ; Subramani, 2004). Aujourd'hui, certaines entreprises cherchent à aller plus loin dans l'intégration du SI de la supply chain, soit par l'intégration des échanges d'informations stratégiques (Klein et Rai., 2009), soit par l'utilisation d'applications plus récentes tels des modules SCM et/ou CRM intégrés à l'ERP, désormais communément appelés ERP II (Beatty et Williams, 2006 ; Koh et al., 2008). Ces nouvelles applications voient leur implémentation encore plus difficile du fait de leur nature collaborative (Koh et al., 2011).

Globalement, la littérature en SI promeut largement l'idée que l'intégration du SI est une condition nécessaire à l'entreprise étendue pour supporter l'intégration de sa chaîne logistique (Bensaou et Venkatraman, 1995; Truman, 2000). Si elle ressort majoritairement de la littérature, cette proposition est parfois contestée. Ainsi, Su et Yang (2010) montrent que dans le secteur Taïwanais de l'informatique et des semi-conducteurs, l'intégration technique réussie et les effets organisationnels positifs ne garantissent pas l'amélioration des compétences logistiques. Ils avancent comme explications possibles d'une part la complexité qu'entraîne la flexibilité dans les opérations et d'autre part les habitudes et le système légal chinois où les usines de Taïwan ont été délocalisées. Cependant, ils montrent aussi que les bénéfices stratégiques et opérationnels de l'ERP le rendent indispensable pour le développement des compétences logistiques. Ainsi, dans la littérature en gestion des opérations, l'idée de Venkatraman domine et s'exprime le plus souvent de la façon simple suivante : l'intégration du SI dans l'entreprise, et notamment par les ERP, conditionne le développement de l'intégration du SI de la supply chain (Al-Mudimigh et al., 2004 ; 
Gunasekaran et Ngai, 2004 ; Overby et Min, 2001; Swafford et al., 2008). Cependant, cette hypothèse n'a jamais été testée dans l'ensemble de l'économie sur un large échantillon. Si ce résultat semble valide dans quelques secteurs (assurances, électronique et informatique) et sur des études de cas, nous nous proposons de tester cette hypothèse au plus près de son origine sur la base de données représentatives des entreprises en France. La figure 2 représente l'hypothèse centrale de la recherche. Avant de présenter la méthodologie mise en œuvre, nous identifions au préalable dans la partie suivante les facteurs d'influence de l'intégration des SI qui seront par la suite testés. Il s'agira en effet de mener des comparaisons entre les facteurs d'influence de l'intégration interne du SI et ceux de l'intégration du SI de la chaîne logistique et de vérifier le lien de causalité entre les deux concepts d'intégration en tenant compte de ces facteurs d'influence.

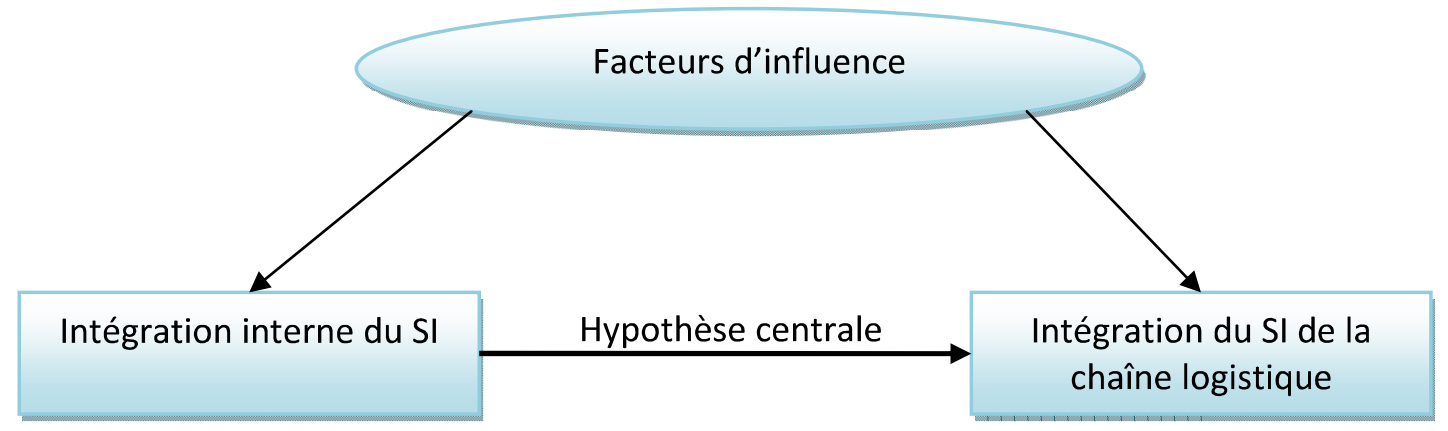

Figure 2 : représentation de l'hypothèse centrale de la recherche

\subsection{Les facteurs d'influence}

La littérature permet d'identifier potentiellement de nombreux facteurs de contingence tant pour l'intégration interne du SI en que pour l'intégration du SI de la supply chain. Nous nous limiterons ici aux facteurs proposés par l'enquête, qui incorpore les plus classiques. Un apport central de la recherche est alors de tester le lien entre les deux concepts d'intégration en tenant compte de ces facteurs d'influence.

La taille de l'entreprise est ainsi un facteur classique à prendre en considération pour discuter de l'informatisation (Goode et Gregor, 2008). Des enquêtes sectorielles montrent en général une corrélation entre l'intégration et la taille de l'entreprise (Lee et Xia, 2006). Le secteur économique est également un élément important en raison d'effets endogènes de l'informatisation (Benzoni et al., 1991). Depuis Stigler (1951), on sait que la division du travail est limitée par la taille du marché et donc, en suivant la conception de Mintzberg (1982), que la taille du marché conditionne les besoins de coordination. Il s'ensuit que les systèmes d'information servant les besoins de coordination dépendent de l'étendue géographique des marchés.

Selon Venkatraman (1994) et une grande part de la littérature en SI, on ne peut passer de l'intégration interne des SI à l'intégration externe sans redéfinir l'organisation. Cela peut se faire concrètement sur différents plans : restructuration financière, refonte de la structure formelle et de l'organigramme, redéploiement d'unités sur le territoire. Sur ce dernier plan, la constitution des SI va de pair et est particulièrement sensible avec l'internationalisation 
(implantation à l'étranger ou délocalisation) dans la mesure où il est à la fois indispensable de se coordonner à distance, mais où les solutions intégrées sont parfois plus difficiles à mettre en œuvre.

Dans le modèle classique de Bensaou et Venkatraman (1995) et dans les théories de la contingence, l'incertitude de l'environnement est un facteur important du fit qui peut influencer l'intégration. Il comprend aussi bien l'évolution des normes réglementaires, de la technologie, l'incertitude du marché, celle concernant les variations des taux de change et celle liée à l'apparition de nouveau concurrents. Le système de finalisation de l'entreprise définit les buts et missions prioritaires, notamment en termes de produits, prestations ou services. Il s'agit notamment de mettre l'accent sur leur variété, leur nouveauté, leur qualité, leur personnalisation ou les prix (Porter, 1985). Dans notre perspective, il est donc intéressant d'examiner dans quelle mesure une politique d'entreprise orientée vers la réduction des délais influe sur les choix d'intégration.

De plus, le système de finalisation s'appuie sur un système de contrôle ou de pilotage dont la littérature montre qu'on peut très schématiquement distinguer deux pôles très différents (Fernandez et al., 1996). D'un côté, des entreprises pratiquent essentiellement un suivi financier en raison d'un manque de moyen ou d'une position dans l'espace concurrentiel qui rend difficile l'anticipation sur les marchés finaux. De l'autre, on trouve celles qui mettent en place des outils de prévision et contrôlent les écarts avec les réalisations afin de se repositionner et développent ainsi une forme plus poussée d'apprentissage organisationnel par les outils de gestion. Enfin, le degré d'importance de l'ensemble de ces variables de contingence dépend de l'appartenance à un groupe ou à un réseau que nous devrons aussi utiliser comme variable de contrôle.

\section{Données et méthodologie}

\subsection{L'enquête COI-TIC et l'équipement informatique des entreprises}

Pour tester notre hypothèse, nous nous appuyons sur les questions permettant de traiter de l'intégration que l'on trouve dans l'enquête nationale "Changements organisationnels et technologies de l'information et de la communication » (COI-TIC) réalisée fin 2005-début 2006 par le pôle «Enquêtes structurelles dans les secteurs des services » de la Division Régionale de l'INSEE des Pays de la Loire. Cette enquête a été menée auprès d'un large échantillon d'entreprises comprenant au moins 10 salariés des secteurs marchands, ce qui inclut les services financiers et d'assurance ${ }^{1}$. Ayant participé à sa conception, nous avons veillé à inclure les éléments centraux portant sur l'intégration du SI. Les éléments contextuels que nous rangeons dans les facteurs d'influence résultent de discussions collectives multidisciplinaires.

Les questionnaires ont été envoyés par courrier et l'enquête avait un caractère obligatoire, ce qui explique le taux de réponse global à l'enquête de 84\%. Les 13790 entreprises qui ont effectivement répondu au questionnaire en représentent 163099 dans le champ de l'enquête après pondération. Pour chacune d'entre elles, l'enquête indique la présence ou non des

\footnotetext{
${ }^{1}$ Pour une description complète de la méthodologie retenue pour cette enquête, se reporter au numéro spécial de la revue Réseaux (2010, $\left.n^{\circ} 162\right)$ intitulé "Informatisation et changements organisationnels dans les entreprises".

Voir aussi le site COI : http://www.enquetecoi.net/
} 
fonctions suivantes : 1) conception, 2) achats - approvisionnement, 3) vente - distribution, 4) production - exploitation, 5) informatique - système d'information, 6) ressources humaines formation, 7) comptabilité - finance - contrôle de gestion. D'après l'enquête COI-TIC, 62,6\% des firmes sont caractérisées par la présence des 7 fonctions et 22,3\% par 6 fonctions. Afin de raisonner à périmètre constant des firmes, nous choisissons pour la suite de l'étude de conserver uniquement les firmes intégrant l'ensemble des fonctions à l'exception de la fonction informatique. Notre échantillon comprend au final 9721 firmes qui représentent 102615 entreprises après pondération ${ }^{2}$. Pour chaque entreprise interrogée, l'enquête comporte plusieurs questions sur l'utilisation des systèmes d'information dans l'entreprise. Le tableau 1 décrit les applications informatiques utilisées suivant les fonctions.

Tableau 1. Les applications informatiques utilisées, par fonction dans l'entreprise

\begin{tabular}{|l|l|l|l|l|}
\hline Fonction & $\begin{array}{l}\text { Fonction } \\
\text { informatisée }\end{array}$ & PGI-ERP & Autre progiciel & $\begin{array}{l}\text { Application } \\
\text { maison }\end{array}$ \\
\hline 1. Conception & $56,9 \%$ & $5,6 \%$ & $29,9 \%$ & $23,9 \%$ \\
2. Achats & $65,4 \%$ & $12,0 \%$ & $22,1 \%$ & $32,6 \%$ \\
3. Vente & $74,4 \%$ & $12,0 \%$ & $29,0 \%$ & $35,4 \%$ \\
4. Production & $68,5 \%$ & $10,2 \%$ & $25,1 \%$ & $35,5 \%$ \\
6. Ressources humaines & $55,0 \%$ & $5,7 \%$ & $26,3 \%$ & $24,4 \%$ \\
7. Comptabilité-finance & $92,5 \%$ & $14,1 \%$ & $57,0 \%$ & $25,5 \%$ \\
\hline
\end{tabular}

Source : enquête COI-TIC, données pondérées.

Note: la somme en ligne des pourcentages pour les outils PGI-ERP, autre progiciel et application maison n'est pas égale à 100 puisque plusieurs outils peuvent être utilisés lorsque la fonction existe dans l'entreprise.

Deux résultats principaux apparaissent. D'un côté, comme l'indique la deuxième colonne du tableau 1, nous observons des différences substantielles dans l'informatisation des fonctions. Si l'usage d'applications informatiques pour la fonction comptabilité-finance est présent dans $92,5 \%$ des cas, il descend à environ $55 \%$ pour les fonctions ressources humaines et conception. De l'autre, il existe également de larges variations dans le type d'application informatique mobilisée. L'utilisation d'un PGI-ERP est plus fréquente pour la comptabilité finance $(14,1 \%)$, les achats $(12 \%)$ et la vente $(12 \%)$. Les autres progiciels du marché concernent surtout la comptabilité - finance, dans 57\% des cas. Quant aux applications maison, elles sont plus souvent utilisées pour la production $(35,5 \%)$, la vente $(35,4 \%)$ et les achats $(32,6 \%)$. L'informatique apparaît donc largement répandue dans l'entreprise. Après pondération, seules $5 \%$ des firmes n'ont aucune fonction informatisée. Elles sont à l'inverse $32 \%$ à avoir 6 fonctions informatisées et plus de la moitié des firmes $(51,4 \%)$ ont au moins 4 fonctions informatisées. De plus, concernant les ERP, seulement 1,7\% des entreprises les utilisent pour toutes leurs fonctions tandis que $79.8 \%$ d'entre elles n'en utilisent aucun.

D'autres questions de l'enquête apportent des éléments complémentaires sur l'intégration des systèmes d'information dans l'entreprise. Différents items (applications utilisées pour les différentes fonctions, outils d'interfaçage, bases de données centrales) sont autant d'éléments constitutifs de l'intégration du SI dans l'entreprise. S'il est aisé d'étudier la diffusion de chacun des outils considérés de façon isolée, il s'avère en revanche beaucoup plus délicat de comparer les entreprises entre elles dans leur usage du système d'information. Le problème

\footnotetext{
${ }^{2}$ Le fait d'inclure la fonction informatique dans le cumul des fonctions ne modifie qu'à la marge l'échantillon (et en conséquence les résultats obtenus par la suite). Seulement 23 firmes ont l'ensemble des fonctions à l'exception de l'informatique. Les exclure n'apparaît toutefois pas approprié dans la mesure où ces entreprises qui n'ont pas de fonction informatique peuvent avoir un recours moins grand aux applications informatiques, du fait de l'absence de la fonction.
} 
majeur vient de l'impossibilité de comparer les outils et applications a priori. En l'absence d'hypothèses a priori, comment savoir par exemple si l'intégration du SI est plus importante lorsqu'une entreprise utilise des logiciels maison pour quatre de ses fonctions par rapport à une entreprise utilisant des progiciels pour deux ou trois de ses fonctions ? Ou bien comment prendre en compte l'incidence respective d'outils d'interfaçage de bases de données relativement à l'informatisation de telle ou telle fonction ? La question centrale est donc celle de l'agrégation de ces différentes variables relatives à l'intégration du SI dans l'entreprise. Plutôt que de postuler a priori des poids pour chacun des outils et applications (avec toutes les chances de commettre des erreurs d'appréciation pour les pondérations), nous proposons à présent une méthode fondée sur une analyse en composantes principales qui permet d'obtenir un indicateur synthétique de l'intégration du système d'information en interne.

\subsection{Construction d'un indicateur d'intégration interne du SI}

\subsubsection{Méthodologie de construction de l'indicateur synthétique}

L'analyse en composantes principales (ACP) est particulièrement appropriée pour construire un indicateur synthétique (Filmer et Pritchett, 2001 ; Vyas et Kumaranayake, 2006). Pour la présentation, nous supposons que nous avons un ensemble de $K$ variables $X_{k, i}(k=1, \ldots, K)$ qui sont corrélées avec l'intégration du SI dans l'entreprise, avec $\mathrm{i}=1, \ldots, \mathrm{N}$ désignant l'entreprise. Notant $\bar{X}_{k}$ la moyenne et $\sigma_{k}$ l'écart-type de la variable $\mathrm{X}_{\mathrm{k}, \mathrm{i}}$, le principe de l'ACP consiste à exprimer chacune des variables normalisées $\widetilde{\mathrm{X}}_{\mathrm{k}, \mathrm{i}}=\left(\mathrm{X}_{\mathrm{k}, \mathrm{i}}-\overline{\mathrm{X}}_{\mathrm{k}}\right) / \sigma_{\mathrm{k}}$ sous la forme d'une combinaison linéaire d'un ensemble de composantes principales $C_{1}, \ldots, C_{K}$. Par inversion, celles-ci peuvent donc s'écrire sous la forme :

$$
\left\{\begin{array}{l}
\mathrm{C}_{1, \mathrm{i}}=\delta_{11} \widetilde{\mathrm{X}}_{1, \mathrm{i}}+\delta_{12} \widetilde{\mathrm{X}}_{2, \mathrm{i}}+\cdots+\delta_{1 \mathrm{~K}} \widetilde{\mathrm{X}}_{\mathrm{K}, \mathrm{i}} \\
\mathrm{C}_{\mathrm{K}, \mathrm{i}}=\delta_{\mathrm{K} 1} \widetilde{\mathrm{X}}_{1, \mathrm{i}}+\delta_{\mathrm{K} 2} \widetilde{\mathrm{X}}_{2, \mathrm{i}}+\cdots+\delta_{\mathrm{KK}} \widetilde{\mathrm{X}}_{\mathrm{K}, \mathrm{i}}
\end{array}\right.
$$

Pour chaque composante principale J, les paramètres $\delta_{\mathrm{jk}}(\mathrm{j}=1, \ldots, \mathrm{K})$ sont les poids associés aux $\mathrm{K}$ variables retenues $\widetilde{\mathrm{X}}_{\mathrm{k}, \mathrm{i}}$ pour rendre compte de l'intégration du SI dans l'entreprise. Ils correspondent en fait aux valeurs propres de la matrice de corrélation entre les différentes variables normalisées $\widetilde{\mathrm{X}}_{\mathrm{k}, \mathrm{i}}$. Ces composantes principales sont ordonnées telles que $\mathrm{C}_{1}>\mathrm{C}_{2}>$ $\ldots>\mathrm{C}_{\mathrm{K}}$. Ainsi, $\mathrm{C}_{1}$ explique la variance maximale des données sous la contrainte $\delta_{11}^{2}+\ldots+$ $\delta_{1 \mathrm{~K}}^{2}=1$. La composante $\mathrm{C}_{2}$, qui est orthogonale à $\mathrm{C}_{1}$, explique ensuite une proportion supplémentaire, mais moins importante de l'information contenue dans les données. Nous retenons dès lors l'indicateur synthétique suivant pour chaque entreprise $\mathrm{i}=1, \ldots, \mathrm{N}$ :

$$
\mathrm{C}_{1, \mathrm{i}}-\sum_{\mathrm{k}} \delta_{1 \mathrm{k}} \widetilde{\mathrm{X}}_{\mathrm{k}, \mathrm{i}}
$$

Par définition, cet indicateur est centré. Des valeurs élevées vont rendre compte d'une intégration interne du SI plus importante. L'hypothèse centrale pour la construction de cet indicateur est que l'intégration du SI explique la variance maximale des variables indiquant l'utilisation des différents outils et applications informatiques. D'un point de vue pratique, nous retenons 29 variables muettes pour construire notre indicateur synthétique (cf Tableau 
2). Pour chaque fonction, 4 dummies permettent de repérer l'utilisation d'un ERP, d'un progiciel du marché, d'un logiciel maison ou l'absence d'informatisation. Les 5 autres dummies indiquent la présence d'outils d'interfaçage de bases de données et celle de bases de données centrales pour 4 fonctions distinctes (conception, vente, ressources humaines, comptabilité). D'après les données, la proportion de la covariance expliquée par la première composante principale est de $18,7 \%$, la valeur propre associée étant de 5,43.

Dans la mesure où les variables constitutives de l'indicateur sont binaires, une variable $\mathrm{X}_{\mathrm{k}}$ caractérisée par un facteur de score $\delta_{1 \mathrm{k}}$ positif conduit à accroître le degré d'intégration interne du SI (et inversement lorsque $\delta_{1 \mathrm{k}}<0$ ). Les ratios $\delta_{1 \mathrm{k}} / \sigma_{\mathrm{k}}$ ont par ailleurs une interprétation simple. Pour une variable $\mathrm{X}_{\mathrm{k}}$ donnée, le passage d'une valeur de 0 à 1 conduit à modifier l'indicateur synthétique de $\delta_{1 \mathrm{k}} / \sigma_{\mathrm{k}}$.

\subsubsection{ACP et validation de l'indicateur synthétique d'intégration interne du SI}

Les résultats du tableau 2 permettent alors de repérer les facteurs qui influencent le plus le degré d'intégration du SI et donc ainsi d'apprécier la qualité de notre indicateur synthétique. Tout d'abord, la présence d'un ERP accroît sensiblement l'intégration et ceci quelle que soit la fonction considérée. Avoir un ERP pour une fonction donnée de l'entreprise accroît en moyenne l'indicateur synthétique de 0,6-0,7. Ensuite, le rôle des progiciels et applications maison est moins clairement défini. Par exemple, pour la conception, les deux types d'application ont une incidence positive sur l'indicateur d'intégration, avec un impact presque trois fois plus élevé pour les progiciels $(0,179$ au lieu de 0,063$)$. Pour les achats, la vente et la production, les scores associés aux progiciels et applications maison sont négatifs, mais de faible ampleur. La présence de progiciels joue positivement pour la fonction ressources humaines, tandis que l'intégration tend à diminuer en présence de progiciels ou d'applications maison pour la fonction comptabilité.

Tableau 2. Facteurs de score et ACP - intégration interne du SI

\begin{tabular}{|c|c|c|c|c|c|}
\hline Variables & & Facteur de score & Moyenne & Ecart-type & Score/écart-type \\
\hline \multirow[t]{4}{*}{ 1. Conception } & PGI - ERP & 0,187 & 0,056 & 0,231 & 0,663 \\
\hline & Autre progiciel & 0,084 & 0,299 & 0,458 & 0,179 \\
\hline & Application maison & 0,028 & 0,239 & 0,427 & 0,063 \\
\hline & Non informatisée & $-0,170$ & 0,431 & 0,495 & $-0,352$ \\
\hline \multirow[t]{4}{*}{ 2. Achats } & PGI - ERP & 0,327 & 0,120 & 0,325 & 0,754 \\
\hline & Autre progiciel & $-0,031$ & 0,221 & 0,415 & $-0,074$ \\
\hline & Application maison & $-0,054$ & 0,326 & 0,469 & $-0,116$ \\
\hline & Non informatisée & $-0,217$ & 0,346 & 0,476 & $-0,513$ \\
\hline \multirow[t]{4}{*}{ 3. Vente } & PGI - ERP & 0,314 & 0,120 & 0,324 & 0,742 \\
\hline & Autre progiciel & $-0,046$ & 0,290 & 0,454 & $-0,103$ \\
\hline & Application maison & $-0,046$ & 0,354 & 0,478 & $-0,096$ \\
\hline & Non informatisée & $-0,192$ & 0,256 & 0,437 & $-0,505$ \\
\hline \multirow[t]{4}{*}{ 4. Production } & PGI - ERP & 0,312 & 0,102 & 0,303 & 0,756 \\
\hline & Autre progiciel & $-0,024$ & 0,251 & 0,434 & $-0,055$ \\
\hline & Application maison & $-0,039$ & 0,355 & 0,478 & $-0,080$ \\
\hline & Non informatisée & $-0,194$ & 0,315 & 0,464 & $-0,496$ \\
\hline \multirow[t]{4}{*}{ 6. $\mathrm{RH}$} & PGI - ERP & 0,227 & 0,057 & 0,232 & 0,669 \\
\hline & Autre progiciel & 0,103 & 0,263 & 0,440 & 0,210 \\
\hline & Application maison & $-0,023$ & 0,244 & 0,429 & $-0,054$ \\
\hline & Non informatisée & $-0,227$ & 0,450 & 0,498 & $-0,511$ \\
\hline \multirow[t]{3}{*}{ 7. Comptabilité } & PGI - ERP & 0,312 & 0,141 & 0,348 & 0,689 \\
\hline & Autre progiciel & $-0,139$ & 0,570 & 0,495 & $-0,278$ \\
\hline & Application maison & $-0,067$ & 0,255 & 0,436 & $-0,159$ \\
\hline
\end{tabular}




\begin{tabular}{|l|l|l|l|l|}
\multicolumn{1}{|c|}{ Non informatisée } & $-0,123$ & 0,075 & 0,264 & $-0,667$ \\
Outils d'interfaçage de BDD & 0,177 & 0,223 & 0,416 & 0,368 \\
BDD centrales - conception & 0,217 & 0,279 & 0,448 & 0,442 \\
BDD centrales - vente & 0,236 & 0,419 & 0,493 & 0,480 \\
BDD centrales - RH & 0,235 & 0,280 & 0,449 & 0,470 \\
BDD centrales - comptabilité & 0,232 & 0,481 & 0,500 & 0,499 \\
\hline Indicateur d'intégration interne du SI & & 0,000 & 2,329 & \\
\hline
\end{tabular}

Source : enquête COI-TIC, données pondérées.

Les différentes valeurs obtenues pour les facteurs de score suggèrent au total que le recours à une ACP permet d'obtenir un indicateur d'intégration du système d'information pertinent. Dans le souci de valider notre démarche, nous avons réalisé deux analyses complémentaires destinées à montrer la qualité de notre indicateur. Dans un premier temps, nous calculons le niveau moyen de l'indicateur d'intégration en fonction du nombre de fonctions informatisées dans les entreprises. On s'attend a priori à ce que la moyenne de l'indicateur synthétique soit d'autant plus élevée que l'usage des applications informatiques concerne un grand nombre de fonctions. Cette hypothèse est pleinement vérifiée d'après les résultats présentés dans la figure 3 .

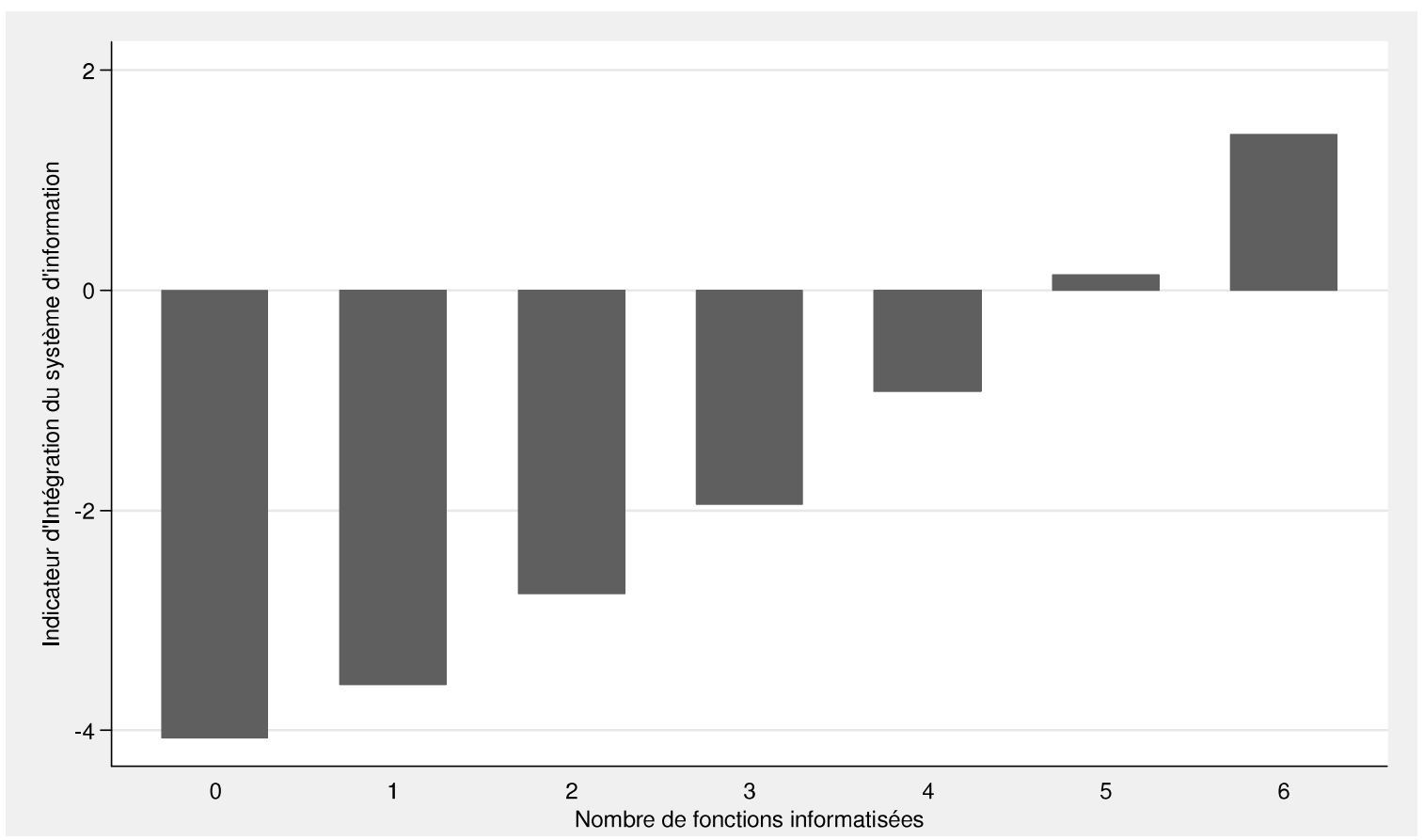

Figure 3. Indicateur synthétique d'intégration interne du SI et nombre de fonctions informatisées

Source : enquête COI-TIC, données pondérées.

Dans un second temps, nous avons ordonné les entreprises suivant la valeur de l'indicateur synthétique. A priori, celles qui ont des valeurs de l'indicateur synthétique élevées doivent davantage utiliser des applications informatiques et des bases de données. Nous avons alors choisi de classer les entreprises suivant quatre quartiles. Le tableau A en annexe décrit la présence des systèmes d'information en fonction de l'appartenance aux différents quartiles de l'indicateur synthétique. Les résultats que nous obtenons attestent de la pertinence de l'indicateur agrégé. Si l'on considère par exemple les fonctions non informatisées, celles-ci s'observent bien plus souvent dans les firmes caractérisées par de faibles valeurs de l'indicateur. De manière similaire, le taux d'usage des ERP est beaucoup plus élevé pour les 
entreprises qui ont des scores synthétiques d'intégration élevés, tandis que les ERP ne sont quasiment jamais présents dans les firmes du premier quartile.

\subsection{Construction d'un indicateur d'intégration du SI de la supply chain}

Suivant la méthodologie décrite précédemment (voir 3.3.1), nous avons ensuite construit un indicateur d'intégration du SI de la supply chain. Nous mobilisons à cet effet les questions suivantes qui ont été posées dans l'enquête COI-TIC. Tout d'abord, on sait si l'entreprise dispose d'un extranet $(31,5 \%)$ et utilise l'EDI ou d'autres liaisons informatiques spécifiques $(40,5 \%)$. Ensuite, l'entreprise peut mobiliser des dispositifs logistiques qui peuvent être soit des outils de traçabilité (des produits, des processus, RFID, etc.), soit des outils/logiciels d'optimisation de la chaîne logistique. L'enquête indique également si l'entreprise utilise un système informatisé de gestion des ventes ou achats, si elle est couplée à des systèmes informatiques qui peuvent être soit un système de gestion des approvisionnements, soit un système de gestion de la production, de l'exploitation ou de la livraison, soit un système de gestion de la facturation et des paiements. Les données précisent aussi si l'entreprise reçoit des commandes de biens ou services par voie électronique, soit par Internet, soit via l'EDI ou d'autres réseaux informatiques (n'utilisant pas Internet comme support), si elle passe des commandes de biens ou services par voie électronique, soit par Internet, soit via l'EDI ou d'autres réseaux informatiques (n'utilisant pas Internet comme support), si le plus gros client dispose d'un système couplé avec l'entreprise (commandes, facturation) et enfin si le plus gros fournisseur dispose d'un système couplé avec l'entreprise (commandes, facturation).

L'application d'une ACP aux données issues des 13 variables retenues nous permet alors de construire un indicateur synthétique d'intégration du SI de la supply chain. Le tableau 3 précise le rôle des différents facteurs d'intégration retenus sur l'indicateur synthétique. La présence de logiciels d'optimisation de la chaîne logistique et les différents couplage avec le système de gestion des ventes ou des achats lorsqu'il est informatisé accroît l'indicateur de 0,7 en moyenne. L'intégration du SI de la supply chain est aussi sensiblement plus forte quand les commandes sont passées ou reçues par l'EDI ou d'autres réseaux informatiques ${ }^{3}$.

Tableau 3. Facteurs de score et ACP - intégration du SI de la supply chain

\begin{tabular}{|c|c|c|c|c|}
\hline Variables & $\begin{array}{l}\text { Facteur de } \\
\text { score }\end{array}$ & Moyenne & Ecart-type & $\begin{array}{l}\text { Score/écart- } \\
\text { type }\end{array}$ \\
\hline Extranet & 0,174 & 0,233 & 0,422 & 0,359 \\
\hline EDI et autres liaisons spécifiques & 0,289 & 0,323 & 0,468 & 0,579 \\
\hline Outils de traçabilité & 0,287 & 0,267 & 0,442 & 0,584 \\
\hline Logiciel d'optimisation de la chaîne logistique & 0,303 & 0,124 & 0,330 & 0,710 \\
\hline Système informatisé de gestion ventes/achats & & & & \\
\hline Couplage système approvisionnement & 0,370 & 0,333 & 0,471 & 0,743 \\
\hline Couplage système production exploitation & 0,379 & 0,353 & 0,478 & 0,759 \\
\hline Couplage système facturation & 0,341 & 0,497 & 0,500 & 0,698 \\
\hline Commandes reçues via Internet & 0,144 & 0,182 & 0,386 & 0,350 \\
\hline Commandes reçues via EDI et autres réseaux & 0,334 & 0,091 & 0,287 & 0,847 \\
\hline Commandes passées via Internet & 0,137 & 0,271 & 0,445 & 0,297 \\
\hline Commandes passées via EDI et autres réseaux & 0,271 & 0,055 & 0,227 & 0,858 \\
\hline Système info couplé avec le plus gros client & 0,234 & 0,095 & 0,293 & 0,638 \\
\hline
\end{tabular}

\footnotetext{
${ }^{3}$ La robustesse de l'indicateur d'intégration externe est étudiée dans le tableau B en annexe. Les différents éléments d'intégration sont tous beaucoup plus présents dès lors que l'on considère des valeurs importantes de l'indicateur.
} 
Système info couplé avec le plus gros fournisseur

0,190

0,135

0,000

0,341
0,501

Source : enquête COI-TIC, données pondérées.

\section{Analyse économétrique de l'intégration}

Pour la présentation des résultats, nous rendons compte tout d'abord des facteurs d'influence de l'intégration avant de traiter des liens unissant l'intégration interne du SI et l'intégration du SI de la supply chain pour tester notre hypothèse centrale de recherche.

\subsection{Les facteurs d'influence de l'intégration}

Dans cette section, nous cherchons à expliciter les déterminants de plusieurs variables dépendantes mesurant l'intégration du SI en tenant compte des deux indicateurs synthétiques d'intégration construits précédemment. Les résultats des régressions linéaires de type Moindres Carrés Ordinaires sont présentés dans le tableau 4.

Tableau 4. Les déterminants de l'intégration du système d'information

\begin{tabular}{|c|c|c|c|}
\hline \multicolumn{2}{|l|}{ Variables } & $\begin{array}{l}\text { Indicateur d'intégration } \\
\text { interne du SI }\end{array}$ & $\begin{array}{l}\text { Indicateur d'intégration du } \\
\text { SI de la supply chain }\end{array}$ \\
\hline \multirow{10}{*}{$\begin{array}{l}\text { Constante } \\
\text { Secteur d'activité }\end{array}$} & & $-3.462^{* * *}$ & $-2.236 * * *$ \\
\hline & Industries agro-alimentaires & 0.112 & $1.347^{* * *}$ \\
\hline & Biens de consommation & $0.173 * *$ & $0.687^{* * *}$ \\
\hline & Biens d'équipement & $0.827^{* * *}$ & $0.466 * * *$ \\
\hline & Biens intermédiaires & $0.687^{* * *}$ & $0.622^{* * *}$ \\
\hline & Construction & 0.026 & $-0.338 * * *$ \\
\hline & Commerce & $0.166 * *$ & $1.011^{* * *}$ \\
\hline & Transport & $-0.431 * * *$ & $0.395 * * *$ \\
\hline & Finance et immobilier & $0.252 * * *$ & $-0.640 * * *$ \\
\hline & Services aux entreprises & Ref & Ref \\
\hline \multirow[t]{5}{*}{ Taille } & 10-19 salariés & Ref & Ref \\
\hline & 20-49 salariés & $0.329 * * *$ & $0.110 * *$ \\
\hline & 50-249 salariés & $1.140 * * *$ & $0.371 * * *$ \\
\hline & 250-499 salariés & $1.783^{* * *}$ & $0.688^{* * *}$ \\
\hline & 500+ salariés & $2.160 * * *$ & $0.978 * * *$ \\
\hline \multicolumn{2}{|c|}{ Appartenance à un groupe } & $0.544 * * *$ & $0.326 * * *$ \\
\hline \multicolumn{2}{|c|}{ Appartenance à un réseau } & 0.046 & $0.325 * * *$ \\
\hline \multirow[t]{4}{*}{ Marché couvert } & Marché local/régional & Ref & Ref \\
\hline & National & $0.226 * * *$ & $0.127^{* * *}$ \\
\hline & Européen & $0.205^{* * *}$ & $0.355^{* * *}$ \\
\hline & International (hors UE) & $0.688 * * *$ & $0.353^{* * *}$ \\
\hline \multicolumn{2}{|c|}{ Restructuration financière depuis 2003} & 0.041 & -0.034 \\
\hline \multicolumn{2}{|c|}{ Refonte de l'organigramme depuis 2003} & $0.295^{* * *}$ & $0.056^{*}$ \\
\hline \multicolumn{2}{|c|}{ Implantation à l'étranger/délocalisation depuis 2003} & $0.412^{* * *}$ & $0.389 * * *$ \\
\hline \multicolumn{2}{|c|}{ Incertitude de l'environnement } & $0.024 * * *$ & $0.014^{* *}$ \\
\hline \multirow[t]{4}{*}{ Reporting } & Aucun & Ref & Ref \\
\hline & Rentabilité financière seulement & $0.264 * * *$ & 0.092 \\
\hline & Pilotage de l'activité seulement & $0.241 * *$ & $0.240^{* * *}$ \\
\hline & Rentabilité et pilotage & $0.814^{* * *}$ & $0.379 * * *$ \\
\hline \multirow[t]{5}{*}{ Importance stratégique } & Variété & 0.012 & 0.012 \\
\hline & Nouveauté & $0.230 * * *$ & $0.154 * * *$ \\
\hline & Qualité & -0.014 & 0.032 \\
\hline & Prix compétitifs & -0.036 & $0.047^{* *}$ \\
\hline & Personnalisation & $-0.039^{*}$ & $-0.075^{* * *}$ \\
\hline
\end{tabular}




\begin{tabular}{|c|c|c|}
\hline $\begin{array}{l}\text { Réduction des délais } \\
\text { Nombre de fonctions informatisées } \\
\text { Nombre de PGI-ERP } \\
\text { Nombre de progiciels } \\
\text { Nombre de logiciels maison }\end{array}$ & $0.417^{* * *}$ & $\begin{array}{l}0.419 * * * \\
0.165 * * * \\
0.225 * * * \\
0.087 * * * \\
0.042 * * *\end{array}$ \\
\hline $\begin{array}{l}\text { Nombre d'entreprises } \\
\text { Pseudo } R^{2}-R^{2}\end{array}$ & $\begin{array}{l}9721 \\
0.403 \\
\end{array}$ & $\begin{array}{l}9721 \\
0.470\end{array}$ \\
\hline
\end{tabular}

Source : enquête COI-TIC, données pondérées.

Note : les estimateurs sont obtenus à partir de modèles linéaires de type MCO. Les seuils de significativité retenus sont de $1 \%(* * *), 5 \%(* *)$ et $10 \%(*)$. Les t de Student associés ne sont pas reportés

\subsubsection{Les facteurs d'influence de l'intégration interne du SI}

Les résultats des facteurs d'influence de l'intégration interne du SI sont reportés dans la seconde colonne du tableau 4. Nous y constatons tout d'abord des différences significatives pour les secteurs d'activité. Ainsi, les deux secteurs de biens d'équipement et intermédiaires proposent l'indicateur d'intégration le plus élevé : une augmentation de 0,83 pour les biens d'équipement et de 0,69 pour les biens intermédiaires par rapport aux services aux entreprises considérés comme catégorie de référence. A l'inverse, dans le transport, l'indicateur d'intégration est significativement plus faible $(-0,43)$. De plus, l'effet de la taille joue très fortement. Par rapport aux entreprises de 10-19 salariés, la valeur de l'indicateur augmente de 0,33 pour les entreprises de 20 à 49 salariés, 1,14 pour les entreprises de 50 à 249 salariés, 1,78 pour celles qui ont $250-499$ salariés et enfin 2,16 pour les plus grandes entreprises.

Si l'appartenance à un groupe a une influence significative positive sur l'indicateur synthétique d'intégration du SI dans l'entreprise, l'appartenance à un réseau ne le modifie pas significativement. Concernant le marché couvert, on voit que plus la couverture est large, plus le SI en interne est intégré : ainsi, l'indicateur synthétique de l'intégration interne du SI augmente sa valeur de 0,69 pour un marché à l'international par rapport à un marché local. Pour les transformations organisationnelles, il apparaît que la restructuration financière n'influence pas significativement l'intégration interne du SI, contrairement à la restructuration de l'organigramme $(+0,30)$ et à l'implantation à l'étranger $(+0,41)$. L'incertitude de l'environnement a une influence significative très faible $(+0,02)$. De plus, l'intégration interne du SI est positivement influencée par la mise en place de reporting sur la rentabilité $(+0,26)$ et le pilotage $(+0,24)$, mais cela est surtout vrai quand les deux types de reporting sont couplés $(+0,81)$. Enfin, concernant l'importance stratégique, la variété, la qualité, la compétitivité des prix et la personnalisation n'ont pas une influence significative importante sur l'intégration interne du SI, contrairement à la nouveauté et surtout à la réduction des délais.

\subsubsection{Les facteurs d'influence de l'intégration du SI de la supply chain}

Les déterminants de l'intégration du SI de la supply chain sont étudiés dans la dernière colonne du tableau 4. Les résultats mettent en évidence une forte disparité de l'intégration du SI de la supply chain selon le secteur d'activité et la taille des entreprises. D'une part, il y a près de 2 points d'écart dans le score d'intégration du SI de la supply chain entre les industries agro-alimentaires et le secteur finance et immobilier. Ce dernier est le secteur le moins intégré au niveau de sa supply chain, suivi de la construction et des services aux entreprises. D'autre part, l'intégration du SI de la supply chain augmente avec la taille de l'entreprise. L'indicateur synthétique est bien plus élevé pour les entreprises de 250 à 499 
salariés $(+1,06)$ et de plus de 500 salariés $(+1,44)$ par rapport à celles ayant de 10 à 19 salariés.

Concernant les autres facteurs, des similitudes sont constatées entre leur influence sur l'intégration du SI de la supply chain et leur influence sur l'intégration interne du SI. Ainsi, nous retrouvons une influence positive de l'appartenance à un groupe, de la taille du marché et du reporting sur l'intégration du SI de la supply chain. Concernant l'importance stratégique, comme pour l'indicateur d'intégration interne du SI, seules la nouveauté et la réduction des délais ont une influence significative positive sur l'intégration du SI de la supply chain. Par contre, nous constatons aussi certaines différences importantes de l'influence de quelques facteurs sur les deux indicateurs : l'appartenance à un réseau influence significativement l'indicateur d'intégration du SI de la supply chain $(+0,33)$, contrairement à la refonte de l'organigramme.

Déjà intégrés dans la construction de l'indicateur synthétique du SI en interne, nous n'avions pas tenu compte de l'influence des éléments importants de l'informatisation (nombre de fonctions, de PGI-ERP, de progiciels spécialisés et de logiciels maison) sur l'indicateur synthétique de l'intégration interne du SI. Pour l'indicateur synthétique de l'intégration du SI de la supply chain, nous les avons cette fois considérés. Conformément aux attentes, ceux-ci sont corrélés positivement avec le degré d'intégration du SI de la supply chain. Le nombre de fonctions informatisées $(+0,165)$ et le nombre d'ERP $(+0,225)$ expliquent davantage l'intégration du SI de la supply chain que le nombre de progiciels dédiés $(+0,087)$ ou le nombre d'applications maison $(+0,042)$.

\subsubsection{Synthèse sur les facteurs d'influence de l'intégration}

La comparaison des estimateurs obtenus pour les indicateurs du SI en interne et du SI de la supply chain révèle que globalement les facteurs d'influence n'ont pas exactement le même effet sur les deux concepts d'intégration. Pour analyser plus finement nos deux indicateurs d'intégration, nous les comparons désormais à travers le rôle de la taille des entreprises et des secteurs d'activité, qui sont ressortis comme les deux principaux facteurs d'influence (voir scores du tableau 4). Dans le premier cas, la figure 4 montre que l'effet de la taille de l'entreprise joue de façon très symétrique pour les deux indicateurs synthétiques d'intégration (avec un trend fortement croissant). Dans le second cas, il existe en revanche des différences significatives par secteur en termes de scores. Les résultats pour le commerce et la finance sont sur ce point très révélateurs. En effet, l'indicateur synthétique moyen de l'intégration interne du SI est négatif pour le commerce, alors que celui de la supply chain est positif. Ainsi, l'intégration interne du SI est relativement faible pour le commerce alors que l'intégration du SI de la supply chain est plutôt bien développée. Le cas du secteur finance et immobilier est diamétralement opposé. C'est cette fois l'indicateur de l'intégration du SI de la supply chain qui est négatif quand celui de l'interne est positif. Les scores moyens d'intégration sont de même signe pour tous les autres secteurs (voir figure 5). 


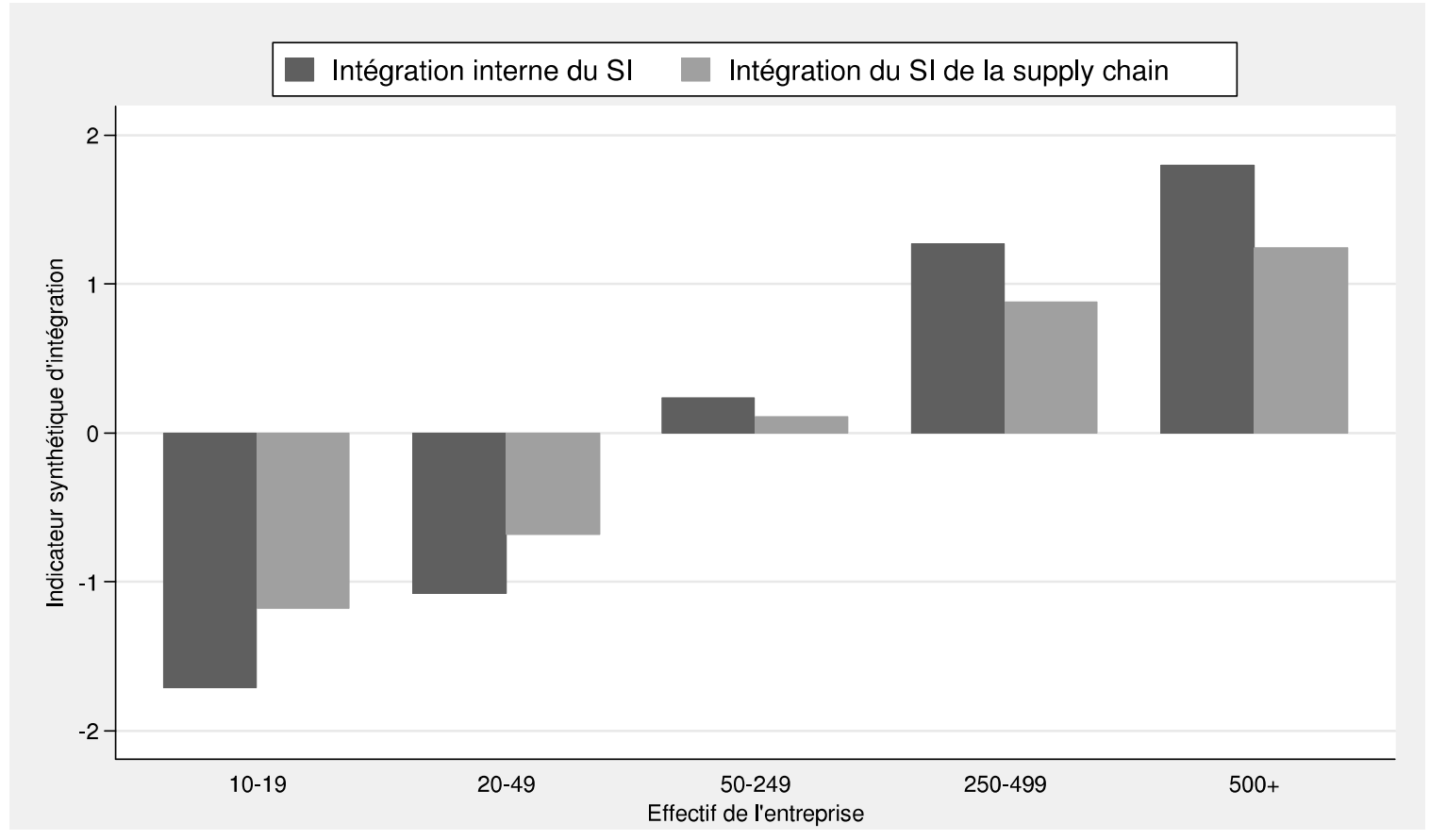

Figure 4. Effets comparés de la taille des entreprises sur l'intégration

Source : enquête COI-TIC, données pondérées.

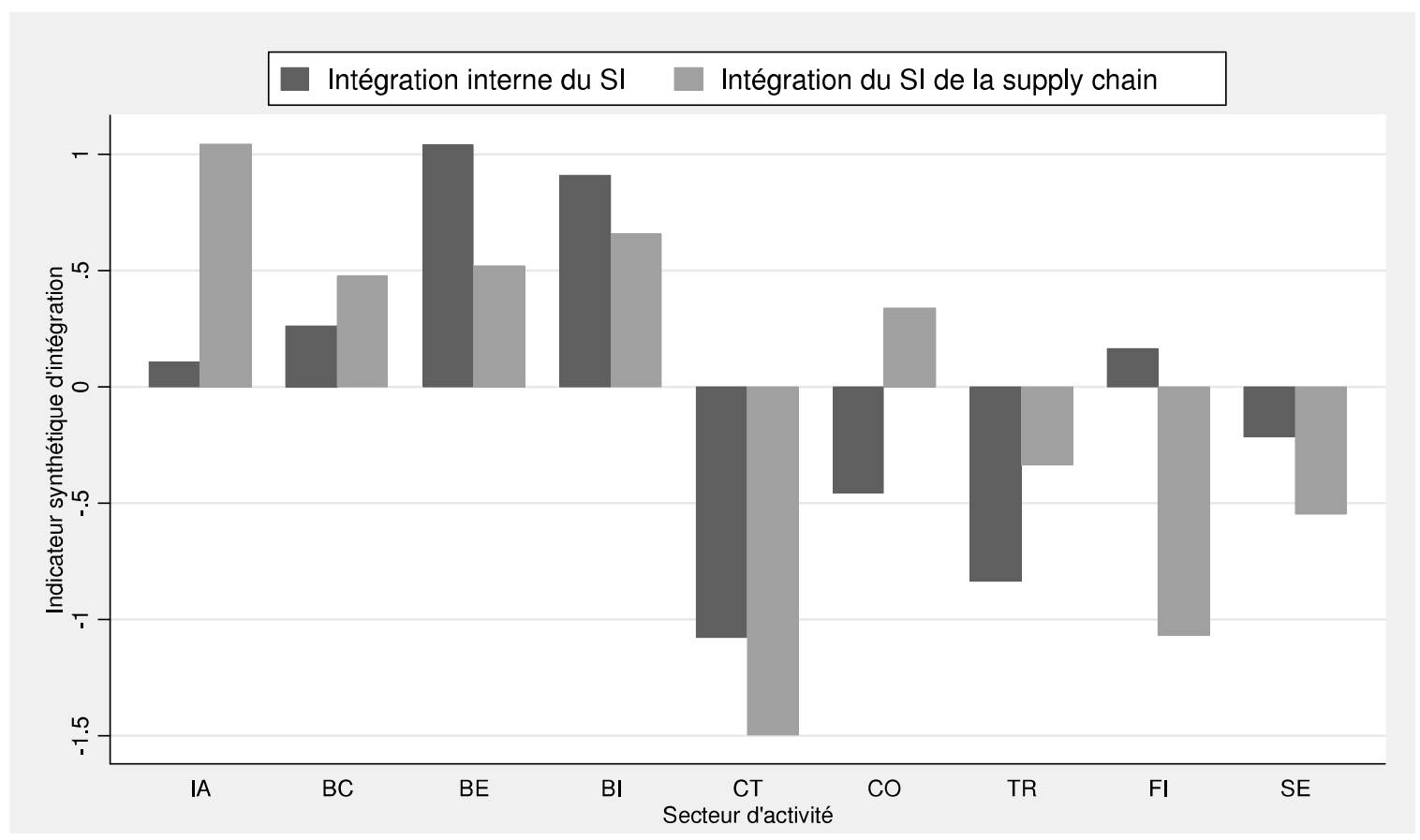

Figure 5. Effets comparés des secteurs d'activité sur l'intégration

Source : enquête COI-TIC, données pondérées.

Légende $: \mathrm{IA}=$ industries agro-alimentaires, $\mathrm{BC}=$ biens de consommation, $\mathrm{BE}=$ biens d'équipement, $\mathrm{BI}=$ biens intermédiaires, $\mathrm{CT}=$ construction, $\mathrm{CO}=$ commerce, $\mathrm{TR}=$ transport, $\mathrm{FI}=$ finance et immobilier, $\mathrm{SE}=$ service aux entreprises.

La figure 6 reprend le cadre de la figure 2 en y spécifiant les facteurs d'influence de l'intégration du SI en interne et de celui de la supply chain. Nous y distinguons les facteurs 
qui ont une influence significative sur les deux indicateurs d'intégration, et ceux influençant uniquement un des indicateurs.

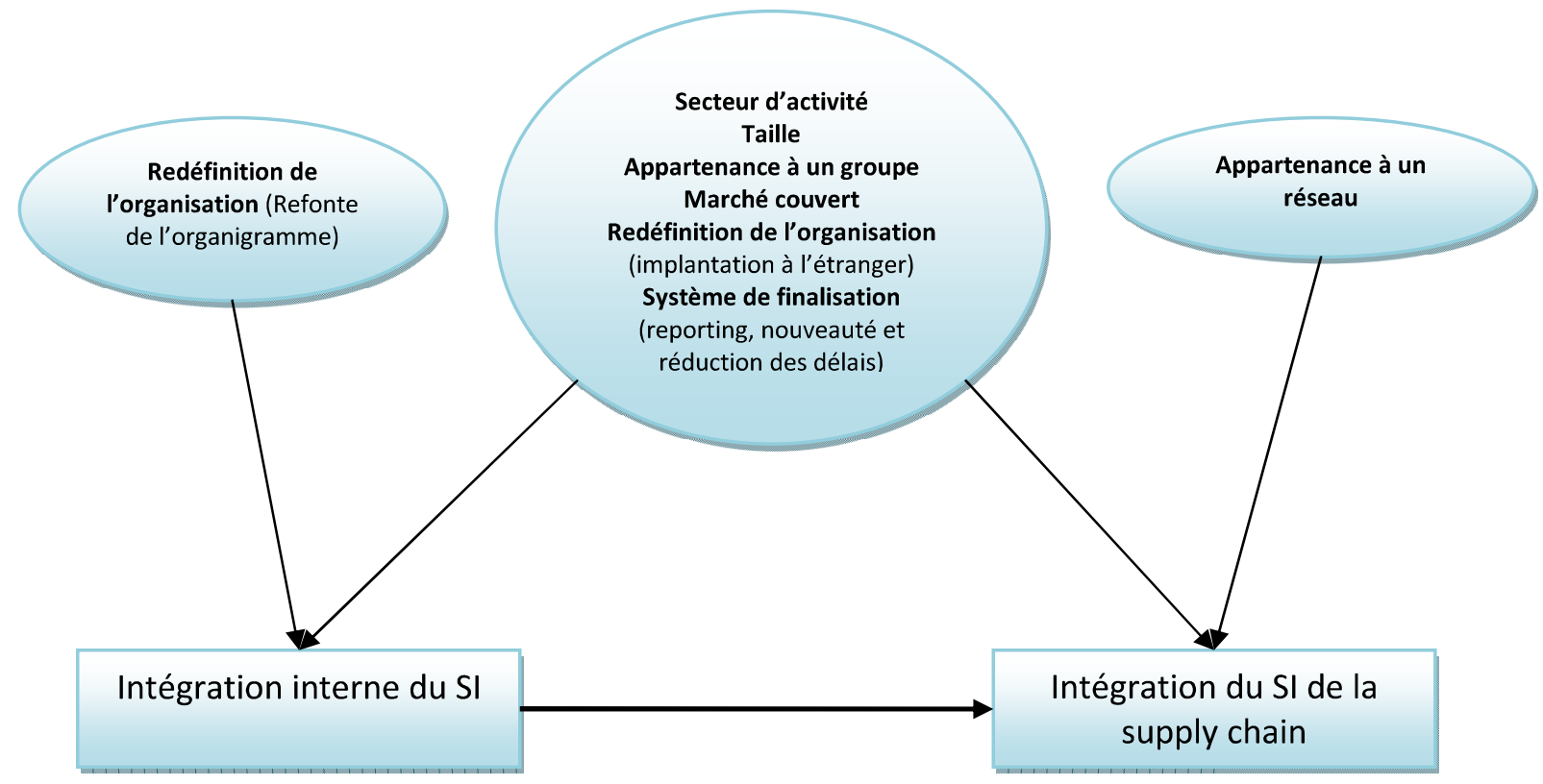

Figure 6 : représentation des facteurs d'influence de l'intégration des SI

De plus, comme spécifié à la fin de la précédente section, les éléments importants de l'informatisation (nombre de fonctions, de PGI-ERP, de progiciels spécialisés et de logiciels maison) sont corrélés positivement avec le degré d'intégration du SI de la supply chain. Ceci constitue un premier élément qui va dans le sens de notre hypothèse centrale de recherche. Dans la section suivante, nous approfondissons par l'analyse économétrique la question du lien entre intégration interne du SI et intégration du SI de la supply chain, tant sur la corrélation que la causalité.

\subsection{Les liens entre l'intégration interne du SI et l'intégration du SI de la supply chain}

Nous essayons ici de mieux comprendre l'interaction entre les deux concepts de l'intégration. Nous présentons ainsi d'abord les résultats sur la corrélation entre les deux concepts de l'intégration du SI et sur la robustesse de la corrélation aux différents facteurs d'influence. La seconde section pose enfin l'analyse économétrique de la causalité.

\subsubsection{La corrélation entre les deux concepts de l'intégration du SI}

Le coefficient de corrélation linéaire est égal à 0,511 d'après les données et il est significatif au seuil de $1 \%$. Il existe donc une forte corrélation positive entre l'intégration interne du SI et l'intégration du SI de la supply chain pour l'ensemble des entreprises en France de plus de 10 salariés. Il est intéressant de noter que cette corrélation positive est observée pour tous les secteurs d'activité. Néanmoins, comme le montre la figure 7, il subsiste des différences sectorielles dans l'intensité de cette corrélation. Elle est particulièrement élevée pour les biens de consommation, les biens d'équipements, les industries agro-alimentaires et les biens intermédiaires. A l'inverse, la corrélation est sensiblement moins forte dans le secteur de la finance et de l'immobilier et dans une moindre mesure pour le transport. La nature des 
transactions en jeu explique a priori la plus faible interaction entre l'intégration interne et l'intégration externe. A la nécessité d'avoir de très fortes connexions en interne pour permettre la totalité des opérations en jeu, la nécessité d'une sécurisation avec l'extérieur est évidente.

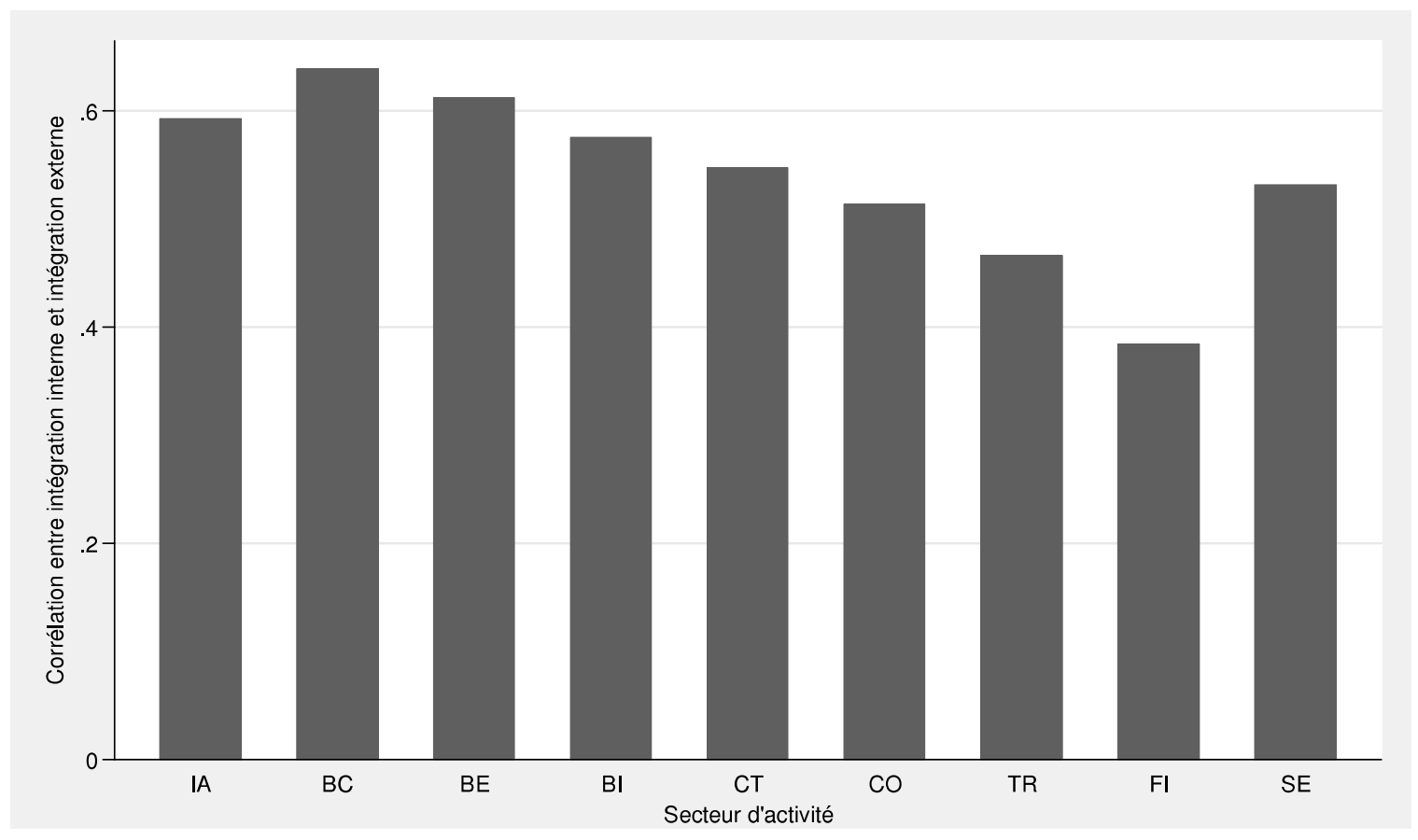

Figure 7. Effets comparés des secteurs d'activité sur l'intégration

Source : enquête COI-TIC, données pondérées.

Légende $: \mathrm{IA}=$ industries agro-alimentaires, $\mathrm{BC}=$ biens de consommation, $\mathrm{BE}=$ biens d'équipement, $\mathrm{BI}=$ biens intermédiaires, $\mathrm{CT}=$ construction, $\mathrm{CO}=$ commerce, $\mathrm{TR}=$ transport, $\mathrm{FI}=$ finance et immobilier, $\mathrm{SE}=$ service aux entreprises.

Pour apporter un éclairage à la question de la robustesse de la corrélation, nous estimons tout d'abord par un modèle linéaire de type MCO le score d'intégration du SI de la supply chain en fonction $\mathrm{du}$ score d'intégration interne du SI. En oubliant l'hétérogénéité liée aux caractéristiques des firmes, les estimations reportées dans le tableau 5 (colonne 1) confirment la corrélation positive entre les deux concepts de l'intégration analysés. Il importe toutefois de prendre en compte le rôle joué par les différents facteurs d'influence (colonne 2). Ceux-ci viennent sensiblement réduire le rôle joué par l'indicateur synthétique du SI en interne, le coefficient associé diminuant d'environ $40 \%$ (0.275 au lieu de 0.450). La corrélation entre l'intégration des SI, en interne et de la supply chain, reste toutefois positive et significative.

Tableau 5. L'effet de l'intégration interne du SI sur l'intégration du SI de la supply chain

\begin{tabular}{|c|c|c|c|c|c|c|c|c|}
\hline Variables explicatives & $\mathrm{MCO}$ & MCO & IV & IV & MCO & MCO & CEM & CEM \\
\hline $\begin{array}{l}\text { Indicateur d'intégration interne } \\
\text { Quintiles d'intégration interne } \\
\text { Facteurs d'influence }\end{array}$ & $\begin{array}{l}0.450 * * * \\
(65.15)\end{array}$ & $\begin{array}{l}0.275^{* * *} \\
(35.56)\end{array}$ & $\begin{array}{l}0.469 * * * \\
(62.58)\end{array}$ & $\begin{array}{l}0.293 * * * \\
(33.23)\end{array}$ & $\begin{array}{l}0.721^{* * *} \\
(62.54) \\
\text { NON }\end{array}$ & $\begin{array}{l}0.429 * * * \\
(33.65) \\
\text { OUI }\end{array}$ & $\begin{array}{l}0.664^{* * *} \\
(47.25) \\
\text { NON }\end{array}$ & $\begin{array}{l}0.426 * * * \\
(29.10) \\
\text { OUI }\end{array}$ \\
\hline $\begin{array}{l}\text { Nombre d'observations } \\
R^{2}\end{array}$ & $\begin{array}{l}9721 \\
0.304\end{array}$ & $\begin{array}{l}9721 \\
0.481\end{array}$ & $\begin{array}{l}9671 \\
0.303\end{array}$ & $\begin{array}{l}9671 \\
0.481\end{array}$ & $\begin{array}{l}9721 \\
0.287\end{array}$ & $\begin{array}{l}9721 \\
0.474\end{array}$ & $\begin{array}{l}6489 \\
0.256\end{array}$ & $\begin{array}{l}6489 \\
0.454\end{array}$ \\
\hline
\end{tabular}

Source : enquête COI-TIC, données pondérées. 
Note : les estimateurs sont obtenus à partir de modèles linéaires de type MCO. Les seuils de significativité retenus sont de $1 \%(* * *), 5 \%(* *)$ et $10 \%(*)$, les t de Student ne sont pas reportés.

\subsubsection{La causalité entre les deux concepts de l'intégration du SI}

Pour traiter de la causalité, nous considérons tout d'abord une stratégie de type variable instrumentale (IV). Dans notre contexte, un instrument est une variable fortement corrélée avec l'indicateur d'intégration interne du SI, mais n'ayant pas d'incidence directe sur l'intégration du SI de la supply chain (l'instrument ne peut avoir qu'un effet indirect, à travers son rôle sur l'intégration du SI en interne). Il est a priori très difficile de penser à une variable corrélée à un seul des deux concepts de l'intégration. Nous avons ici choisi de retenir pour instrument une valeur retardée de l'intégration interne du SI. Pour tous les items retenus pour cet indicateur synthétique, des informations sur la situation à la fois en 2006 et en 2003 sont disponibles dans le questionnaire. Nous avons donc eu recours à une analyse en composantes principales pour obtenir un indicateur synthétique d'intégration interne du SI à la date de 2003. Cette variable apparaît alors très fortement corrélée avec le score interne courant (avec un $\mathrm{R}^{2}$ partiel proche de 0,8). L'estimation par les Doubles Moindres Carrés (colonnes 3 et 4), qui doit permettre de corriger les biais d'endogénéité et donc de mesurer l'effet causal de l'intégration interne du SI sur l'intégration du SI de la supply chain, ne modifie pas réellement les coefficients obtenus par les MCO. L'intégration du SI de la supply chain apparait plus forte quand l'intégration interne du SI est elle-même importante, la relation étant très fortement significative. Néanmoins, la validité de l'instrument peut poser question dans la mesure où l'indicateur interne construit pour 2003 a de fortes chances d'être lui-même corrélé avec l'indicateur d'intégration de la supply chain de 2003. Il peut alors y avoir un problème de variable omise puisque l'intégration du SI de la supply chain à cette date est susceptible de fortement conditionner l'intégration en 2006.

Une technique alternative consiste alors à passer par des techniques d'appariement, qui permettent de tenir compte de la sélection sur observables. Nous appliquons à cet effet la méthode CEM (Coarsened Exact Matching) développée par Blackwell et al. (2009). L'idée est la suivante. Les valeurs du score d'intégration interne du SI peuvent être vues comme un traitement. Pour déterminer l'effet causal de cette variable sur l'intégration du SI de la supply chain, il faut tenir compte des différences antérieures au traitement (par exemple les grandes firmes ont une intégration du SI plus forte que les autres) de telle sorte que ces écarts soient réduits au minimum sur la base de certaines caractéristiques observées pour les firmes ${ }^{4}$. En pratique, le traitement pour la technique CEM ne peut être une variable continue. Nous construisons donc une variable ordonnée correspondant aux quintiles du score d'intégration interne. Les colonnes 5 et 6 du tableau 5 montrent que l'utilisation de cette variable ordonnée ne modifie pas nos conclusions précédentes. L'effet des quintiles de l'indicateur d'intégration interne sur l'intégration du SI de la supply chain est positif et diminue sensiblement une fois les facteurs d'influence pris en compte. L'appariement CEM se fait ensuite sur la base des caractéristiques suivantes pour les firmes : secteurs d'activité, taille, appartenance à un groupe, à un réseau et couverture de marché ${ }^{5}$. De nouveau, nous trouvons bien que l'intégration interne du SI vient accroître l'intégration du SI de la supply chain (colonnes 7 et 8).

\footnotetext{
${ }^{4}$ Les propriétés et avantage de la technique CEM sont discutés en détail par lacus et al. (2011).

${ }^{5}$ Nous limitons volontairement le nombre de caractéristiques pour l'appariement en ne retenant que les principaux facteurs d'influence. La multiplication des variables de contrôle réduit au fur et à mesure l'échantillon apparié, qui combine des firmes identiques sur la base de leur quintile d'intégration interne et de leurs caractéristiques sélectionnées.
} 


\section{Discussion}

Sur la base de données précisant l'usage des SI dans les entreprises françaises et à l'aide d'outils économétriques, notre étude empirique nous conduit à valider notre hypothèse centrale, basée sur l'approche de Venkatraman (1994), selon laquelle l'informatisation de l'entreprise étendue est positivement corrélée à l'informatisation interne de l'entreprise. Par la construction d'indicateurs synthétiques sur l'intégration interne du SI et l'intégration du SI de la supply chain, nous avons notamment montré qu'intégration interne et externe allaient de pair, quels que soient la taille ou le secteur d'activité de l'entreprise. De plus, la mobilisation de techniques d'appariement et par variable instrumentale (Blackwell et al., 2009) suggère l'existence d'un lien de causalité entre les deux concepts d'intégration. Ainsi, une forte intégration interne du SI favorise l'intégration du SI de la supply chain. Cet article valide donc l'hypothèse souvent avancée de la corrélation et la causalité entre intégration interne du SI et intégration du SI de la supply chain, et ce sur un large échantillon des entreprises françaises de plus de 10 salariés incorporant toutes les fonctions classiques.

De manière plus fine, l'analyse met en évidence que le nombre et le type de technologies intégratives utilisées en interne influencent de manière différente l'intégration, tant interne qu'externe. Il est notamment clair que l'intégration par les ERP est corrélée avec l'intégration du système d'information de la supply chain, confirmant ainsi sur l'ensemble de l'économie française un résultat jusqu'ici très partiel et surtout avancé dans la littérature de gestion des opérations (Al-Mudimigh et al., 2004 ; Gunasekaran et Ngai, 2004 ; Overby et Min, 2001; Swafford et al., 2008).

Nous retrouvons et confirmons aussi le rôle de plusieurs variables de contingence influençant l'intégration du SI des entreprises, tant sur que le plan interne qu'externe. C'est notamment le cas du type de marché, des systèmes de finalisation et de contrôle/pilotage. Il est par ailleurs particulièrement intéressant de relever que l'incertitude de l'environnement, si présente dans la théorie de la contingence, a une influence relativement faible sur l'intégration du SI relativement aux autres variables. Globalement, les facteurs influençant l'intégration interne du SI et ceux influençant l'intégration du SI de la supply chain, sont relativement semblables, à quelques exceptions près.

D'une part, les réorganisations internes, testées notamment par la refonte de l'organigramme, ont une influence quasiment nulle sur l'intégration du SI de la supply chain alors qu'elles sont loin d'être négligeables concernant l'intégration interne du SI. Cela peut s'expliquer par le fait que les transformations organisationnelles à notre époque vont de pair avec une recherche de transversalité dans l'entreprise qui se traduit par la mise en place de technologies intégratives (El Amrani et al., 2006). Cette explication est notamment confirmée par le fait que la refonte de l'organigramme n'est corrélée qu'au nombre d'ERP et non de progiciels spécialisés ou applications maisons. L'ERP, bien plus que les autres progiciels/logiciels, impose de repenser l'organisation en profondeur par une refonte de la gestion des données et des processus (Markus, 2000 ; Davenport, 1998).

D'autre part, l'appartenance à un réseau n'influence que l'intégration du SI de la supply chain. Ce résultat somme toute logique met en exergue que l'intégration du SI de la supply chain est davantage soumise aux relations interorganisationnelles que l'intégration interne du SI. Pour chaque entreprise, les bénéfices issus de l'utilisation de systèmes d'information 
interorganisationnels devraient être fortement dépendants de la masse critique de partenaires y participant (Riggins et Mukhopadhyay, 1994 ; Subramani, 2004). Ainsi, confiance et pouvoir (Hart et Saunders, 1997) sont plus à même d'être mobilisés par des entreprises pour promouvoir l'adoption et l'utilisation de technologies intégratives interorganisationnelles par leurs partenaires quand le réseau d'appartenance est important et formalisé.

Enfin, nos résultats sur la corrélation et la causalité entre l'intégration interne du SI et l'intégration du SI de la supply chain et sur leurs facteurs d'influence sont issus d'une analyse macroscopique de l'état de l'économie française à une date donnée. Ainsi, nous ne pouvons entrer dans la compréhension fine des avantages et inconvénients des systèmes d'intégration pour les entreprises qui pourraient expliquer le schéma d'évolution de l'entreprise proposé par Venkatraman (1994). En particulier, les transformations au niveau des interdépendances et des différenciations induites par une intégration interne (Gattiker et Goodhue, 2005) ont-elles une influence sur le déploiement de systèmes d'intégration de la chaîne logistique ? Une analyse des non alignements sur les six domaines proposés par Strong et Volkoff (2010) dans l'intégration, tant interne qu'externe, permettrait probablement de mieux comprendre la causalité entre les deux concepts d'intégration.

\section{Conclusion}

Sur la base du schéma d'évolution proposé par Venkatraman, nous avons montré par l'utilisation des données de l'enquête COI-TIC que l'intégration interne du système d'information et l'intégration du système d'information de la supply chain sont fortement corrélées. Le nombre d'ERP présents dans les différentes fonctions de l'entreprise vient accroître tant l'intégration interne du SI que l'intégration du SI de la supply chain, ce qui confirme que la mobilisation de technologies intégratives en interne est aussi liée au développement de l'intégration externe. Nos résultats économétriques mettent en évidence un effet causal de l'intégration interne du SI sur l'intégration du SI de la supply chain, confirmant ainsi sur un large échantillon représentatif une hypothèse souvent avancée dans la littérature. D'un point de vue managérial, cette recherche incite les praticiens à considérer l'intégration interne du SI comme souhaitable pour favoriser l'intégration du SI de la supply chain.

La principale limite de cette recherche réside dans le fait que l'analyse des facteurs d'influence a été circonscrite à ceux proposés dans les questions de l'enquête COI-TIC. Une autre analyse pourrait être menée sur l'influence de facteurs complémentaires de l'intégration des SI. Nous pensons notamment aux caractéristiques de la gestion de projet et aux choix stratégiques de mise en oeuvre des technologies intégratives (El-Amrani et al., 2006), au support apporté par les éditeurs ou les communautés de pratique (Saint-Léger et El-Amrani, 2011), ou encore aux différentes dimensions de l'alignement entre l'entreprise et son SI (Strong et Volkoff, 2010). Enfin, les données datant de 2006, il serait intéressant de réitérer une analyse de même type en tenant compte des dernières avancées dans les récentes technologies intégratives interorganisationnelles. Les technologies telles que les ERP II (Koh et al., 2011) modifient-elles la relation de causalité entre intégration interne du SI et intégration du SI de la supply chain ? L'ajout de modules complémentaires à l'ERP et ouverts sur la relation clients et/ou fournisseurs ne modifie probablement pas la nécessité d'avoir en amont repensé l'intégration interne. Il semble bien que c'est avant tout l'intégration interne qui se déplace sur le terrain des opérations interorganisationnelles (Markus, 2000).

Notre analyse empirique révèle par ailleurs des écarts importants et très différents selon les secteurs. Il serait dès lors intéressant tant pour le milieu académique que pour les praticiens de 
mener des études sectorielles complémentaires afin de mieux comprendre ces écarts sectoriels en termes d'intégration. Centrée sur les données de l'enquête COI-TIC qui confèrent une forte validité externe aux résultats, l'analyse reste circonscrite à l'intégration d'un point de vue technique et appelle à une compréhension plus approfondie de l'intégration à d'autres niveaux. De futures recherches pourraient ainsi se concentrer sur les liens qui unissent l'intégration interne et externe (Truman, 2000) aux niveaux informationnels ou bien organisationnels (Kumar et van Dissel, 1996 ; Clergeau et Rowe, 2005, Volkoff et al., 2005).

\section{Références}

Al-Mudimigh A., Zairi M. et Ahmed A.M. (2004), "Extending the Concept of Supply Chain: the Effective Management of Value Chains", International Journal of Production Economics, vol. 87, n³, p. 309-320.

Beatty R. et Williams C. (2006), "ERPII : best practices for successfully implementing an ERP upgrade", Communications of the ACM, vol. 49, n³, p. 105-109.

Bensaou M. et Venkatraman N. (1995), "Configurations of Interorganizational Relationships: a Comparison between US and Japanese Automakers", Management Science, vol. 41, n 9 , p. 1471- 92.

Benzoni L., Lebart L. et Rowe F. (1991), "Market Dynamics and Technological Segmentation: the Case of the French Professional Telecommunication Market", in Antonelli C. (Ed.), The economics of information networks, North-Holland.

Bidan M. (2004), «Fédération et intégration des applications du système d'Information de Gestion ». Systèmes d'Information et Management, vol. 9, n² 2, p. 5-24.

Bidan M. et Rowe F. (2004), "Urbanization Practices and Strategic Behavior: Openness of Architecture and Enactment in two Medium Sized Companies". $9^{\text {ieme }}$ Colloque de l'Association Information \& Management, Mai, Evry, France.

Blackwell M., Iacus S., King G. et Porro G. (2009), "Coarsened Exact Matching in Stata", Stata Journal, vol. 9, n4, p. 524-46.

Clergeau C. et Rowe F. (2005), «Caractérisation des dispositifs d'interdépendance organisationnelle et mutualisation : le cas des centres d'appels virtuels ", Systèmes d'Information et Management, vol. 10, n`3, p. 93-116.

de Corbière F. et Geffroy-Maronnat B. (2009), «Des formes d'intégration intraorganisationnelle aux formes d'intégration interorganisationnelle: analyse du cas des catalogues électroniques », $16^{\text {ième }}$ Colloque de l'Association Information \& Management, Juin, Marrakech, Maroc.

Daniel E. et White A. (2005), "The Future of Interorganizational System Linkages: Findings of an International Delphi Study", European Journal of Information Systems, vol.14, n², p. $188-203$.

Davenport T. (1998), "Putting the Enterprise in the Enterprise System", Harvard Business Review, n'76, pp. 121-131.

El Amrani R., Rowe F. et Geffroy-Maronnat B. (2006), "The effects of ERP Implementation Strategy on Cross-Functionality", Information Systems Journal, vol. 16, n 1, p. 79-104.

El Amrani R. et Saint Léger G., (2011), «Le pari des centres de compétences dans la stabilisation des organisations en phase post-projet ERP », Systèmes d'Information et Management, vol. 16, $\mathrm{n}^{\circ} 1$, p. 69-112.

Fernandez V., Picory C. et Rowe F. (1996), « Outils de gestion et espaces concurrentiels des $\mathrm{PME} »$, Revue Internationale des PME, vol. 9, n¹, p. 79-102. 
Filmer D. et Pritchett L.H. (2001), "Estimating Wealth Effect without Expenditure Data - or Tears: an Application to Educational Enrollments in States of India", Demography, vol. 38, p. 115-132.

Gattiker T. et Goodhue D. (2005), "What Happens after ERP Implementation: Understanding the Impact of Inter-Dependence and Differentiation on Plant-Level Outcomes", MIS Quarterly, vol. 29, n³, p. 559-585.

Goode S. et Gregor S. (2009), "Rethinking Organisational Size: Meaning, Measurement and Redevelopment", European Journal of Information Systems, vol. 18, n¹, p. 14-25.

Gunasekaran A. et Ngai E. (2004), "Information Systems in Supply Chain Integration and Management", European Journal of Operational Research, vol. 159, n², p. 269-95.

Hart P. et Saunders C.S. (1997), "Power and Trust: Critical Factors in the Adoption and Use of Electronic Data Interchange", Organization Science, vol. 8, n¹, p. 23-42.

Iacus S.M., King G. et Porro G. (2011), "Multivariate Matching Methods that are Monotonic Imbalance Bounding", Journal of the American Statistical Association, vol. 106, p. 345361.

Koh S.C.L., Gunasekaran A. et Rajkumar D. (2008), "ERP II: The involvement, benefits and impediments of collaborative information sharing", International Journal of Production Economics, vol.113, n¹, p. 245-268.

Koh S.C.L., Gunasekaran A. et Goodman T. (2011), "Drivers, barriers and critical success factors for ERPII implementation in supply chains: A critical analysis", Journal of Strategic Information Systems, vol. 20, n 4 , p. 385-402.

Klein, R. et Rai A. (2009), "Interfirm Strategic Information Flows in Logistics Supply Chain Relationships", MIS Quarterly, vol.33, n4, p. 735-762.

Kumar K. et Van Dissel H.G. (1996), "Sustainable Collaboration: Managing Conflict and Cooperation in Interorganizational Systems", MIS Quarterly, vol. 20, n³, p. 279-300.

Livolsi L. et Fabbe-Costes N. (2004), «la centralité des systèmes d'information (SI) dans la fonction logistique », Revue Française de Gestion Industrielle, vol. 23, n 4, p. 27-44.

Lee G., et Xia W. (2006), "Organizational Size and IT Innovation Adoption: A MetaAnalysis“, Information and Management, vol. 43, n8, p. 975-85.

Markus M.L. (2000), "Paradigm Shifts-E-Business and Business/Systems Integration”, Communications of the AIS, vol. 4, article 10.

Mintzberg H. (1982), Structure et dynamique des organisations, Editions d'Organisations : Paris.

Overby J. et Min S. (2001), "International Supply Chain Management in an International Environment", International Marketing Review, vol. 18, n4, p. 392-420.

Patnayakuni R., Rai A. et Seth N. (2006), "Relational Antecedents of Information Flow Integration for Supply Chain Coordination", Journal of Management Information Systems, vol. $23, \mathrm{n}^{\circ} 1$, p. $13-49$.

Prajogo D. et Olhager J. (2012), "Supply chain integration and performance: The effects of long-term relationships, information technology and sharing, and logistics integration", International Journal of Production Economics, vol. 135, $\mathrm{n}^{\circ} 1$, p. 514-522.

Porter M. (1985), Competitive Advantage, The Free Press: New York.

Rai A., Patnayakuni R. et Patnayakuni N. (2006), "Firm Performance Impacts of Digitallyenabled Supply Chain Integration Capabilities", MIS Quarterly, vol. 30, n², p. 225-46.

Reix R., Fallery B., Kalika M. et Rowe F. (2011), Systèmes d'Information et Management des Organisations, $6^{\text {ème }}$ édition, Vuibert, 496 pages.

Riggins F.J. et Mukhopadhyay T. (1994), "Interdependent Benefits from Interorganizational Systems: Opportunities for Business Process Re-engineering", Journal of Management Information Systems, vol. 11, n², p. 37-57.

Seddon P., Calvert C. et Yang S. (2010), "A Multi-Project Model of Key Factors Affecting Organizational Benefits from Enterprise Systems”, MIS Quarterly, vol.34, n², p. 305-328. 
Srinivasan K., Kekre S. et Mukhopadhyay T. (1994), "Impact of Electronic Data Interchange Technology on JIT Shipments", Management Science, vol. 40, n¹0, p. 1291-1304.

Stigler G. (1951), "The Division of Labour is Limited by the Extent of the Market", Journal of Political Economy, vol. 59, p. 185-93

Strong, D. et Volkoff O. (2010), "Understanding Organization-Enterprise System Fit: A Path to Theorizing the Information Technology Artifact", MIS Quarterly, vol. 34, n4, p. 731756.

Su Y.-F. et Yang C. (2010), "Why are Enterprise Resource Planning Systems Indispensable to Supply Chain Management?", European Journal of Operational Research, vol. 203, n 1 , p. 81-94.

Subramani M. (2004), "How do Suppliers benefit from Information Technology Use in Supply Chain Relationships?”, MIS Quarterly, vol. 28, n¹, p. 45-73.

Swafford P., Ghosh S. et Murthy N. (2008), "Achieving Supply Chain Agility through IT Integration and Flexibility", International Journal of Production Economics, vol. 116, n², p. 288-97.

Themistocleous M., Irani Z. et Love P. (2004), "Evaluating the Integration of Supply Chain Information Systems: a Case Study", European Journal of Operational Research, vol. 159, $\mathrm{n}^{\circ} 2$, p. 393-405.

Truman G.E. (2000), "Integration in Electronic Exchange Environments", Journal of Management Information Systems, vol. 17, ${ }^{\circ} 1$, p. 209-44.

Venkatraman N. (1994), "IT-enabled Business Transformation: from Automation to Business Scope Redefinition”, Sloan Management Review, vol. 35, n², p. 73-87.

Volkoff O., Strong D. et Elmes M. (2005), "Understanding enterprise systems-enabled integration", vol. $14, \mathrm{n}^{\circ} 2$, p. 110-120.

Vyas S. et Kumaranayake L. (2006), "Constructing Socio-Economic Status Indices: how to use Principal Components Analysis”, Health Policy and Planning, vol. 21, nº, p. 459-68. 
Annexe.

Tableau A. Utilisation des systèmes d'information et indicateur synthétique d'intégration interne

\begin{tabular}{|c|c|c|c|c|c|}
\hline \multirow[t]{2}{*}{ Variables } & & \multicolumn{4}{|c|}{ Indicateur synthétique d'intégration interne } \\
\hline & & Quartile 1 & Quartile 2 & Quartile 3 & Quartile 4 \\
\hline \multirow[t]{4}{*}{ 1. Conception } & $P G I-E R P$ & 0,007 & 0,020 & 0,069 & 0,350 \\
\hline & Autre progiciel & 0,182 & 0,384 & 0,440 & 0,374 \\
\hline & Application maison & 0,179 & 0,285 & 0,366 & 0,187 \\
\hline & Fonction non informatisée & 0,636 & 0,329 & 0,174 & 0,191 \\
\hline \multirow[t]{4}{*}{ 2. Achats } & $P G I-E R P$ & 0,001 & 0,022 & 0,138 & 0,873 \\
\hline & Autre progiciel & 0,142 & 0,342 & 0,344 & 0,062 \\
\hline & Application maison & 0,262 & 0,455 & 0,447 & 0,084 \\
\hline & Fonction non informatisée & 0,599 & 0,190 & 0,089 & 0,038 \\
\hline \multicolumn{2}{|c|}{ 3. Vente PGI-ERP } & 0,004 & 0,024 & 0,142 & 0,842 \\
\hline & Autre progiciel & 0,231 & 0,411 & 0,372 & 0,100 \\
\hline & Application maison & 0,294 & 0,475 & 0,474 & 0,116 \\
\hline & Fonction non informatisée & 0,476 & 0,101 & 0,046 & 0,024 \\
\hline \multirow[t]{4}{*}{ 4. Production } & PGI - ERP & 0,002 & 0,021 & 0,082 & 0,789 \\
\hline & Autre progiciel & 0,174 & 0,358 & 0,385 & 0,104 \\
\hline & Application maison & 0,282 & 0,473 & 0,493 & 0,151 \\
\hline & Fonction non informatisée & 0,551 & 0,160 & 0,076 & 0,054 \\
\hline \multirow[t]{4}{*}{ 6. $\mathrm{RH}$} & $P G I-E R P$ & 0,000 & 0,012 & 0,051 & 0,431 \\
\hline & Autre progiciel & 0,092 & 0,367 & 0,482 & 0,402 \\
\hline & Application maison & 0,165 & 0,340 & 0,382 & 0,122 \\
\hline & Fonction non informatisée & 0,745 & 0,289 & 0,105 & 0,114 \\
\hline \multirow[t]{4}{*}{ 7. Comptabilité } & $P G I-E R P$ & 0,018 & 0,053 & 0,184 & 0,850 \\
\hline & Autre progiciel & 0,590 & 0,665 & 0,587 & 0,207 \\
\hline & Application maison & 0,265 & 0,297 & 0,276 & 0,063 \\
\hline & Fonction non informatisée & 0,154 & 0,011 & 0,004 & 0,005 \\
\hline \multicolumn{2}{|c|}{ Outils d'interfaçage de bases de données } & 0,078 & 0,241 & 0,438 & 0,481 \\
\hline \multicolumn{2}{|c|}{ Bases de données centrales - conception } & 0,062 & 0,295 & 0,651 & 0,609 \\
\hline \multicolumn{2}{|c|}{ Bases de données centrales - vente } & 0,114 & 0,531 & 0,832 & 0,817 \\
\hline \multicolumn{2}{|c|}{ Bases de données centrales - RH } & 0,031 & 0,284 & 0,738 & 0,650 \\
\hline \multicolumn{2}{|c|}{ Bases de données centrales - comptabilité } & 0,180 & 0,604 & 0,870 & 0,875 \\
\hline
\end{tabular}

Source : enquête COI-TIC, données pondérées.

Tableau B. Utilisation des systèmes d'information dans la chaine logistique et indicateur synthétique d'intégration

\begin{tabular}{|l|l|l|l|l|}
\hline \multirow{2}{*}{ Variables } & \multicolumn{3}{l|}{ Indicateur synthétique d'intégration externe } \\
\cline { 2 - 5 } & Quartile 1 & Quartile 2 & Quartile 3 & Quartile 4 \\
\hline Extranet & 0,103 & 0,240 & 0,350 & 0,476 \\
EDI et autres liaisons spécifiques & 0,122 & 0,278 & 0,475 & 0,882 \\
Outils de traçabilité & 0,063 & 0,244 & 0,432 & 0,763 \\
Logiciel d'optimisation de la chaîne logistique & 0,004 & 0,087 & 0,173 & 0,559 \\
Système informatisé de gestion ventes/achats & & & & \\
$\quad$ Couplage SG approvisionnement & 0,007 & 0,271 & 0,770 & 0,863 \\
$\quad$ Couplage SG production exploitation & 0,004 & 0,331 & 0,768 & 0,923 \\
$\quad$ Couplage SG facturation & 0,101 & 0,629 & 0,886 & 0,947 \\
Commandes reçues via Internet & 0,090 & 0,192 & 0,245 & 0,388 \\
Commandes reçues via EDI et autres réseaux & 0,000 & 0,028 & 0,105 & 0,536 \\
Commandes passées via Internet & 0,153 & 0,311 & 0,356 & 0,462 \\
Commandes passées via EDI et autres réseaux & 0,000 & 0,018 & 0,065 & 0,318 \\
Système info couplé avec le plus gros client & 0,015 & 0,095 & 0,104 & 0,369 \\
Système info couplé avec le plus gros fournisseur & 0,014 & 0,128 & 0,244 & 0,390 \\
\hline
\end{tabular}

Source : enquête COI-TIC, données pondérées. 